\title{
Polymer-Mode-Coupling Theory of Finite-Size-Fluctuation Effects in Entangled Solutions, Melts, and Gels. 2. Comparison with Experiment
}

\section{Matthias Fuchs ${ }^{\dagger}$ and Kenneth S. Schweizer*}

Departments of Materials Science and Engineering and Chemistry and Materials Research Laboratory, University of IIlinois, 1304 West Green Street, Urbana, IIlinois 61801

Received February 20, $1997^{\otimes}$

Konstanzer Online-Publikations-System (KOPS)

URL: http://www.ub.uni-konstanz.de/kops/volltexte/2007/3859/

URN: http://nbn-resolving.de/urn:nbn:de:bsz:352-opus-38599

\begin{abstract}
The predictions of polymer mode coupling theory tor the tinite size corrections to the transport coefficients of entangled polymeric systems are tested in comparison with various experimental data. It is found that quantitative descriptions of the viscosities, $\eta$, dielectric relaxation time, $\tau^{\epsilon}$, and diffusion coefficients, D, of polymer melts can be achieved with two mi croscopic structural fit parameters whose values are in the range expected from independent theoretical or experimental information. An explanation for the (apparent) power law behaviors of $\eta, \tau^{\epsilon}$, and $\mathrm{D}$ in (chemically distinct) melts for intermediate molecular weights as arising from finite size corrections, mainly the self-consistent constraint release mechanism, is given. The variation of tracer dielectric relaxation times from Rouse to reptationlike behavi or upon changes of the matrix molecular weight is analyzed. Self-diffusion and tracer diffusion constants of entangled polymer solutions can be explained as well, if one further parameter of the theory is adjusted. The anomal ous scaling of the tracer diffusion coefficients in semidilute and concentrated polystyrene solutions, $\mathrm{D} \sim \mathrm{N}^{-2.5}$, is predicted to arise due to the spatial correlations of the entangl ement constraints, termed "constraint porosity". Extensions of the theory to polymer tracer diffusion through poly(vinyl methyl ether) and polyacrylamide gels provide an explanation of the observation of anomalously high mol ecular weight scaling exponents in a range where the size of the tracer, $\mathrm{R}_{\mathrm{g}}$, al ready considerably exceeds the gel pore size, $\xi_{g}$.
\end{abstract}

\section{Introduction}

The microscopic polymer mode coupling (PMC) theory connects the dynamics of entangled polymeric systems to the underlying equilibrium liquid structure. This description therefore naturally includes corrections arising from the finite molecular weights of tracer or matrix polymers. In the preceding paper, ${ }^{1}$ referred to as paper I, the PMC predictions with finite size corrections have been worked out for the transport properties of entangled solutions and melts and for polymer tracer motion in gels. In the present paper these results are compared with data from many experiments. As the PMC approach has been detailed in the preceding paper, ${ }^{1}$ it shall be summarized only shortly in this section and then compared to alternative approaches. The resulting formulas of paper I will be referenced by the equation numbers of that paper with a prefix I.

The PMC approach of paper I treats the Rouseentangled crossover problem in a simple interpolative manner. The PMC contributions capturing the entanglement corrections are combined with the Rouse, unentangl ed quantities, see eqs I .52 and I .68 for D, eqs 1.55 and 1.67 for $\eta$, and eqs 1.58 and 1.70 for $\tau^{\epsilon}$. Appreciable deviations from the asymptotes for high molecular weights are therefore not explained by corrections of the Rouse dynamics to the asymptotic, entangled motion. In stark contrast to the contour length fluctuation model of Doi, ${ }^{2,3}$ the repton model of Rubinstein, ${ }^{4}$ and the Rouse-fluctuating-chain-end calculations of O'Connor and Ball, 5 the discrete nature of the tracer chain or its chain ends are not the cause of the deviations from the asymptotic molecular weight scalings within PMC theory.

Finite size corrections to the transport coefficients within PMC theory result from the spatial and temporal

\footnotetext{
† Current address: Physik-Department, Technische Universität München, D-85747 Garching, Germany.

${ }^{\otimes}$ Abstract published in Advance ACS Abstracts, J uly 15, 1997.
}

correlations of the matrix constraints, called entanglements. These constraints are modeled with a priori reasonable assumptions. First, the local density variations enter through a nonhomogeneous compressibility, eq I.45. Second, only a finite amplitude of the density fluctuations, or of the shear stress amplitudes (see section 3.B of paper I), contributes to the elastic mesh formed by the entanglements. This amplitude is modeled in analogy to the entanglement plateau observed by neutron scattering from single pol ymers. ${ }^{6-8}$ Except for an overall (small) amplitude, it is characterized by the entangl ement length, b, eq I.46. These two effects are summarily termed "constraint porosity". Further, the temporal decay of the matrix constraints during the disentanglement step of the matrix, see eqs I.47 and I.48, is considered self-consistently; this process is termed "constraint release mechanism" and provides another parallel channel for the relaxation of the constraining excluded volume forces.

Neglecting the constraint release and constraint porosity effects, PMC theory predicts smooth crossovers from Rouse to entangled dynamics, as can be seen from eqs I.52, I.55, and I.58. Reptation-like molecular weight scaling exponents are found. Still, it is important to note that these results qualitatively differ from the corresponding reptation/tube results in the prediction of nonuniversal asymptotic prefactors. The effects on Markovian transport coefficients of the internal mode Rouse dynamics for finite tracer molecular weights, which are the topic of the contour fluctuation, repton, and chain-end fluctuation model $\mathrm{s}^{2-5}$ for finite $\mathrm{N}$ corrections to the terminal relaxation time, are neglected within the PMC approach of paper I. Recent dielectric measurements of Adachi and co-workers ${ }^{9,10}$ would seem to convincingly support the latter approach. A change in the molecular weight dependence of the end-to-endvector relaxation time of a tracer polymer is found upon changes of the molecular weight of the matrix poly- 
mers;9,10 asymptotic-like scaling, $\tau^{\epsilon} \sim M^{3}$, is observed in immobile matrices of high molecular weight polymers. This effect clearly cannot be explained by single polymer approaches but must result from considerations of the dynamics of the matrix constraints.

The importance of constraint release contributions in the viscosity has also been stressed by a recent simulation which focuses on the configurational rearrangements of the entanglement points. ${ }^{11}$ The consideration of the dynamics of the surrounding matrix is an aspect of PMC theory it shares with the constraint release models of Graessley ${ }^{12}$ and Klein. ${ }^{13,14}$ Whereas, in these two approaches, the diffusion coefficients of the reptative and constraint release dynamics are phenomenologically added, in the PMC approach the effects of both decay channels on the entanglement friction functions depend on length scale and are derived from one set of approximations for the microscopic intermolecular forces, as shown in eqs I.11-I.27 of paper I. Moreover, the argument of self-consistently determining the matrix disentanglement time from the dynamics of a single matrix polymer is not included in the former phenomenological constraint release formulations, whereas in PMC theory it is captured via eqs I.64-I.66. It appears that the plasticization model ${ }^{5}$ or the double reptation formalism of des Cloizeaux, ${ }^{15-18}$ can be viewed as a phenomenological attempt to incorporate these effects. In both models, the decay of the conformational dynamics or stress is accelerated by taking a power of a single polymer correlation function. From the perspective of mode coupling approximations, noninteger powers as advocated in ref 5 seem difficult to motivate microscopically.

In des Cloizeaux's work, additional phenomenol ogical concepts such as "time-dependent reptation" or "a displacement-dependent diffusion constant" lead to the appearance of a new intermediate time scale of rather unclear physical origin. This elaboration of the tube model results in a stronger-than-reptation scaling of the internal relaxation time, which is tightly connected to the anomalous frequency dependence of the preterminal wing of the disentanglement process in the shear modulus. ${ }^{16,17}$ In experimental studies of shear moduli ${ }^{19-21}$ and dielectric spectra, ${ }^{22-24}$ the high-frequency wing of the disentanglement process consistently exhibits a power law frequency dependence, $\mathrm{G}^{\prime \prime}\left(\omega \tau_{\mathrm{D}} \gg\right.$ 1) $\sim \omega^{-x}$ and $\epsilon^{\prime \prime}\left(\omega \tau_{D} \gg 1\right) \sim \omega^{-x^{\prime}}$, with exponents clearly much smaller than the reptation prediction, $\mathrm{x}, \mathrm{x}^{\prime}=0.2-$ $0.3<1 / 2$. In this respect it is an important finding of the dielectric measurements that the initial decay of the disentanglement process is still more rapid, and consequently its high-frequency wing still possesses a more shallow slope than the reptation prediction, even in the limit where an $\mathrm{M}^{3}$ mass scaling of the internal relaxation time is observed. ${ }^{9,10}$ Therefore, we believe a consistent and convincing physical mechanism for the anomalous frequency dependence of the initial decay of the disentanglement process remains to be identified theoretically, and it must be different from the mechanism leading to non-reptation-like scaling of the longest relaxation time or viscosity. In PMC theory, this mechanism is the tracer shape fluctuations, as has been shown in section 3.A of paper I, and will further be discussed in section 3.E where the dielectric measurements of Adachi and co-workers ${ }^{9}$ are analyzed; see al so refs 25-27.

Whereas various different non-self-consistent phenomenological formulations of the constraint release mechanism ${ }^{12-14,25}$ have been considered as extensions of the reptation/tube approach, the constraint porosity effects of the PMC description, captured in e.g., eqs I.68 and 1.69 , have no analog in the phenomenological models for polymer melts and solutions. This follows since these models do not relate the entanglement constraints to the static structure of the polymeric liquid. The measurements of tracer diffusion constants in strongly entangled polymer solutions ${ }^{26-29}$ find anomalously strong molecular weight dependences, e.g., D $\propto$ $\mathrm{N}^{-2.5}$. This rules out the constraint release mechanism as the only source of deviations from the $\mathrm{N}^{-2}$ asymptotic scaling. Within the tube approach only an extension of the repton model, i.e., the numerical cage model of Deutsch and Madden, ${ }^{30}$ claims to predict molecular weight scaling of the diffusion coefficients and viscosities in agreement with the measurements in solution. ${ }^{29}$ However, from this phenomenological model it is not apparent why such an $\mathrm{M}$-scaling is not observed in melts. Moreover, this model would again fail in explaining the $\mathrm{M}^{3}$ scaling of the internal relaxation time in the limit of immobile matrices, as observed by dielectric spectroscopy. 9,10 A similar concern applies to the ideas of Rubinstein and Obukhov ${ }^{31}$ where a close connection of the apparent power laws of the diffusion coefficient and viscosity, $\mathrm{D} \eta \propto \mathrm{R}_{\mathrm{g}}{ }^{2}$, is postulated for solutions but not for melts. Further non-reptation theoretical approaches exist ${ }^{32-38}$ but generally have not been worked out in such detail to allow comparison with the mentioned experimental observations and the issues of finite $\mathrm{N}$ corrections and apparent crossover scaling laws.

The static correlations of the matrix constraints as described by PMC theory naturally generalize to amorphous media, especially gels, which are fractal on intermediate length scales. In the context of polymer motion through fractal media it has been recognized by Muthukumar and Baumgärtner that strong deviations from the reptation-like behavior result when the medium is characterized by a length scale or mesh of the order of the size of the diffusing polymer. ${ }^{39-42}$ The entropic barrier model thus agrees with PMC theory in predicting finite size corrections if the spatial length scale of the surrounding medium is not much smaller than the radius of gyration of the tracer. However, in PMC theory the physically reasonable but difficult to quantify concepts, such as "entropic traps" and "lack of topological correlations" 42 can be avoided, as the tracer dynamics is directly connected to the static structure of the gel and to the tracer-gel interactions. Also, the limits of small and infinitely large tracer sizes and smooth crossovers between regimes are naturally included in the PMC description but not in the entropic barrier model. In the PMC approach, the gel is assumed to exhibit pores of any (mesoscopic) size smaller than the gel length scale, $\xi_{g}$, which therefore determines the range of the spatial structural correlations. If flexible or nonrigid gels structures are considered, then another length characterizing the elastic stresses enters the PMC description, i.e., eqs I.79 and I.80. In contrast with the entropic barrier ${ }^{42}$ considerations and its enthalpic trap generalizations ${ }^{43}$ the PMC description of polymer transport through gels predicts strong finite size corrections to tracer diffusion even if $\mathrm{R}_{\mathrm{g}} \gg \xi_{\mathrm{g}}$.

In the present paper the PMC results of paper I shall be compared to experimental data in order to test the theoretical predictions. A priori estimates of the two nonuniversal parameters determining the finite size 
Table 1. Material Parameters of the Different Polymer Systems Studied ${ }^{f}$

\begin{tabular}{|c|c|c|c|c|c|c|c|c|c|}
\hline & PBD & PBDh & PDMS & PEO & $\mathrm{Pl}$ & PS & PS & PS & PS \\
\hline melt or sol (wt \%) & $\mathrm{m}$ & $\mathrm{m}$ & $\mathrm{m}$ & $\mathrm{m}$ & $\mathrm{m}$ & $\mathrm{m}$ & 13 & 18 & 40 \\
\hline $\mathrm{Me}_{\mathrm{e}}$ & $1.85 \times 10^{3}$ & $1.24 \times 10^{3}$ & $1.2 \times 10^{4}$ & $2.2 \times 10^{3}$ & $5.0 \times 10^{3}$ & $1.8 \times 10^{4}$ & $1.3 \times 10^{5}$ & $9.6 \times 10^{4}$ & $4.23 \times 10^{4}$ \\
\hline $\mathrm{N}_{\mathrm{e}}$ & 34 & $44^{e}$ & 162 & 50 & 74 & 173 & 1250 & 923 & 407 \\
\hline$\alpha$ & $4.9,^{a} 3.3^{b}$ & $4.3^{c}$ & $2.8^{b}$ & $2.6^{\mathrm{b}}$ & $2.2,{ }^{b} 3.5^{d}$ & $2.2^{b}$ & $2.8^{\mathrm{b}} 4.0^{\mathrm{a}}$ & $2.8^{b}$ & $2.8^{\mathrm{b}} 2.5^{\mathrm{a}}$ \\
\hline$\delta$ & 0.01 & 0.03 & 0.0 & 0.05 & 0.05 & 0.05 & 0.4 & 0.3 & 0.4 \\
\hline b $(\AA)$ & 44.4 & 33.9 & 78.6 & 37.5 & 62.0 & 76.5 & & & \\
\hline$\xi_{\rho}(\AA)$ & 2.30 & 1.75 & 4.06 & 1.94 & 3.20 & 3.95 & & & \\
\hline
\end{tabular}

${ }^{a}$ From viscosity. ${ }^{b}$ From diffusion. ${ }^{c}$ From viscosity and diffusion. ${ }^{d}$ From $\tau^{\epsilon}$. e $M_{0}^{P B D h}=28$ used. ${ }^{f}$ Molecular weights of entanglement, $\mathrm{M}_{\mathrm{e}}$, are taken from the experimental studies whose data are analyzed, and entanglement degrees of polymerization, $\mathrm{N}_{\mathrm{e}}$, are calculated from them using monomer repeat masses; 81 entanglement lengths, b, are taken from ref 46 for $\mathrm{T}=413 \mathrm{~K}$. The packing lengths, ${ }^{46} \mathrm{p}$, are identified with the density screening length, $\xi_{\rho} \approx 1 / \rho_{\mathrm{m}} \sigma^{2}$, and are also included. The melt or solution case is indicated by $\mathrm{m}$ or by the weight concentration of the polymeric component. Inverse strength parameters, $\alpha$, and length scale ratios, $\delta$, are determined by the fits shown in Figures 1-4, 7, and 8.

corrections to the PMC dynamics are presented in section 2. Section 3 introduces the experimental systems and studies examples for melt viscosities (sections 3.B and 3.C), melt self-diffusion coefficients (sections 3.C and 3.D), melt tracer diffusion constants (section 3.D), dielectric relaxation times in melts (section 3.E), transport coefficients in solutions (section 3.F), and tracer mobilities in gels (section 4). The paper concludes in section 5 with a summary of the results of this and the preceding paper I and suggestions for future experiments to further test our predictions.

\section{Estimates of Parameters}

The PMC theory of paper I predicts that the nonasymptotic corrections to the dynamics of entangled polymers depend on two material and thermodynamicstate-dependent dimensionless parameters of clear microscopic meaning: $\delta=\xi_{\rho} / \mathrm{b}$ and $\alpha$. The first parameter arises from porosity or correlation effects that weaken the entanglement constraints. It involves two measurable quantities: the polymer density-density screening length and the entanglement mesh length scale ('tube diameter" in reptation theory). Although such length scales can be estimated on the basis of liquid state integral equation theory or via computer simulations, the best source of information is experiment. The second parameter enters the prefactor of the asymptotic scaling of the diffusion constant with $\mathrm{N}$, i.e., $\mathrm{D}=$ $D^{R}\left(N_{e} / N\right) \lambda_{D}{ }^{-1}$, where $D^{R}$ is the bare Rouse value and $\lambda_{D}$ $=32 / 3 \alpha$. Thus, $\alpha$ quantifies an effective crossover degree of polymerization for the asymptotic behavior of $\beta D \xi_{0} \rightarrow N_{D} / N^{2}$ where $N_{D}=(3 \alpha / 32) N_{e}$. A microscopic connection with the mean square force exerted on the probe chain center-of-mass by all the surrounding polymers has been derived: $\lambda_{\mathrm{D}}=32 / 3 \alpha \propto\left\langle|\mathrm{F}|^{2}\right\rangle / \rho_{\mathrm{m}}$, where $\rho_{\mathrm{m}}$ is the segmental number density. Hence, $\alpha$ is inversely proportional to the strength of the constraining forces (entanglements) per unit matrix number density. On the basis of liquid state theory, and the Kirkwood superposition approximation, one can derive ${ }^{44,45}\left\langle|\mathrm{~F}|^{2}\right\rangle / \rho_{\mathrm{m}} \propto\left(\mathrm{g}_{\mathrm{d}} \xi_{\rho}\right)^{2}$, where $g_{\mathrm{d}}$ is the contact value of the interchain segment-segment radial distribution function, which quantifies the local hard core excluded volume forces between chains. Although experimentally accessible in principle (and definitely via simulations), $\left\langle|\mathrm{F}|^{2}\right\rangle$ and $\mathrm{g}_{\mathrm{d}}$ do not appear measurable in practice. Thus, we are forced to make a priori estimates of $\alpha$ based on theoretical input.

In this section, we discuss the available experimental information, and theoretical estimates, of the required microscopic parameters. In section 3 we treat them as adjustable parameters determined by fits to viscosity and/or diffusion constant data of specific polymer solutions and melts. The independent, a priori estimates of this section provide strong constraints on the physically acceptable values these parameters can assume.

We also note that, in principle, the entanglement degree of polymerization, $\mathrm{N}_{\mathrm{e}}$, and the polymer material parameters determining its bare (Rouse) dynamics are also required as input to the theory. However, we always consider ratios of transport coefficients relative to the unentangled Rouse values, $\eta / \eta^{\mathrm{R}}$ and $\mathrm{D} / \mathrm{D}^{\mathrm{R}}\left(\mathrm{N}_{\mathrm{e}}\right)$. Thus, we employ the PMC prediction ${ }^{1}$ that these ratios depend on the degree of polymerization only via $\mathrm{N} / \mathrm{N}_{\mathrm{e}}$, where $\mathrm{N}_{\mathrm{e}}$ is taken from experiment and data are plotted in the standard reduced variable format. Thus, comparison to experiment requires only specifying the two nonuniversal parameters $\delta$ and $\alpha$.

A. Experimental Estimates. Consider first the ratio $\delta=\xi_{\rho} / \mathrm{b}$. In Table 1 we list rheologically-extracted (via the relation $\mathrm{G}_{N}=\rho_{\mathrm{m}} \mathrm{k}_{\mathrm{B}} \mathrm{T}(\sigma / \mathrm{b})^{2}$ ) values for the entangl ement mesh, b, of several systems in the melt. ${ }^{46}$ Values appropriate for solutions containing a fraction $\Phi$ of polymer follow from the known scaling relations: $47,48 \mathrm{~b} \propto \Phi^{-v}$ where $v \approx 0.75$ (good solvents where $\xi_{\rho} \propto$ b and $\sigma \propto \Phi^{-1 / 8}$ ) or $v \approx 2 / 3\left(\Theta\right.$ solvents $\left.^{49}\right)$. Remarkably, these scaling relations appear to apply over a wide polymer concentration regime from semidilute up to the melt. ${ }^{50}$ The density screening length has been measured for only a few polymers, and up to $\Phi \approx 0.3-0.4$ at best. For polystyrene, the average of many experiments yields $47 \xi_{\rho} \approx 2.5 \AA \Phi^{-0.72}$ (good solvents) and $\xi_{\rho}$ $\approx 6 \AA \Phi^{-1.0}$ ( $\Theta$ solvents). Of course, as the melt state is approached, $\Theta$ and good solvents become equivalent, and hence these laws must merge into a single concentrated solution behavior. Elementary dense liquid considerati ons require that such simple scaling laws for the screening length cannot hold up to the melt (where $\xi_{\rho}<\sigma$ ). The screening length (and hence the osmotic pressure) decreases (increases) much more rapidly than in semidilute solution and in a non power law fashion. ${ }^{51-54}$ Some nonuniversality is expected in the concentrated and melt regimes.

Extrapolation of polystyrene semidilute good solvent scal ing laws up to the melt suggests a screening length of roughly $3-5 \AA$, which is expected to be an upper bound. Interestingly, as is seen from the table, this extrapolation is in good accord with a value calculated on the basis of the invariant packing length, ${ }^{46} p=$ $1 / \rho_{\mathrm{m}} \sigma^{2}$. The packing length rigorously emerges as essentially equal to $\xi_{\rho}=(\pi / 3) p$ from the simplest analytic version of PRISM theory based on the idealized Gaussian thread or string models. ${ }^{44,45,51-53,55}$ In any case, for polystyrene an upper bound for the parameter 
$\delta=\xi_{\rho} \mathrm{rh} / \mathrm{b} \approx 0.05$ in the melt can be estimated from the experimental data. It is probably smaller, perhaps by a factor of 2 or 3 . This latter estimate follows from thethread PRISM based connection between the density screening length and the dimensi onless measure of long wavelength density fluctuations: ${ }^{44,45,51-53,55} \mathrm{~S}_{0}=\rho_{\mathrm{m}} \mathrm{k}_{\mathrm{B}} \mathrm{T} \kappa$ $=12\left(\xi_{\rho} / \sigma\right)^{2}$, where $\kappa$ is the isothermal compressibility and $\sigma$ the statistical segment length. From scattering experiments or thermodynamic measurements on dense polymer liquids, one finds that $\mathrm{S}_{0}$ is typically in the range $0.15-0.3$. Thus, for polystyrene with a statistical segment length of $7 \AA$ one obtains $\xi_{\rho} \approx 1 \AA$. On the other hand, perhaps the dynamically-rel evant mesh size for entanglements involves only the backbone mass, which would again result in an effective mesh size of

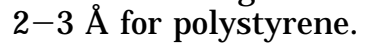

In solution one expects different behaviors depending on solvent qual ity and also chemical structure. In good solvents, where the screening length and entangl ement length are predicted (by scaling ${ }^{48}$ and $\mathrm{PMC}^{44}$ theories) and measured to be proportional, 48,56 one expects a polymer concentration-independent value of $\delta$ but nonuniversality with respect to the chemical structure dependence. The latter failure of naive scaling has been emphasized by Adam and co-workers ${ }^{56}$ who find a correlation of this ratio with the melt $\mathrm{N}_{\mathrm{e}}$. For semidilute good polystyrene solutions, from the arguments given above and known data we expect a larger value of $\delta$ than in the melt, perhaps in the range 0.2-0.3. Recent Monte Carlo simulations ${ }^{57,58}$ of semidilute good polymer solutions of weakly entangled chains find a value of $\delta$ $\approx 0.3$. Even larger values are expected in $\Theta$ solvents since the screening length increases faster than the entanglement length as the polymer concentration is decreased. 47,49,50 Moreover, $\delta$ is expected to be polymer concentration-dependent in $\Theta$ solvents ( $\delta \propto \Phi^{-1 / 3}$ roughly) with increasing values for less concentrated polymer solutions.

On the basis of the above considerations, we have a rather good idea of the magnitude of the key parameter $\delta$ in the melt, and in good and $\Theta$ solutions, at least for polystyrene. Although we expect the concentration dependence of $\delta$ is relatively universal, the magnitude may depend significantly on chemical structure. Direct measurements of the density screening length in other materials is required to determine just how large a variation is possible.

Estimation of the parameter $\alpha$, or equivalently $\lambda_{\mathrm{D}}=$ $32 / 3 \alpha$, from experimental data is more difficult. If the asymptotic scaling regime $\left(D \propto \mathrm{N}^{-2}\right)$ is achieved, then $\alpha$ is immediately determined. However, this appears possible only in melts, and not in solution. The existing experimental data have clearly established that $\lambda_{\mathrm{D}}$ is significantly larger in semidilute and concentrated solutions of polystyrene compared with its melt value. ${ }^{26-29}$

B. Theoretical Estimates. As discussed elsewhere, ${ }^{44,45}$ theoretical estimates of the density screening length, entanglement mesh or $N_{e},\left\langle|F|^{2}\right\rangle / \rho_{m}$, and $g_{d}$ have been made on the basis of a very simple chain model (Gaussian thread or string), analytic PRISM theory, and the RR and PMC dynamical theories. This analysis suggests $\left\langle|\mathrm{F}|^{2}\right\rangle / \rho_{\mathrm{m}}$, or $\alpha$, is nearly polymer concentration and chemical structure independent due to a nearly perfect inverse relationship between the contact value $g_{d}$ and the density screening length. This work provides some first principles justification for polymer density scalings observed experimentally, but quantitatively accurate calculations are difficult. Estimates based on numerical PRISM computations for solutions and melts that employ more chemically realistic chain models (semiflexible Koyama or RIS ${ }^{59}$ ) produce results in surprisingly good semiquantitative agreement with the prior analytic work, even up to melt-like densities where $\mathrm{g}_{\mathrm{d}}{ }^{2} \mathrm{~S}_{0} \approx 0.1-0.2$. Based on eq I.54, we obtain the crude ab initio estimate of $\alpha \approx 2 / g_{d}^{2} S_{0} \approx 10-20$.

At a more detailed level, it is found that $\left\langle|\mathrm{F}|^{2}\right\rangle / \rho_{\mathrm{m}}$ (proportional to $\alpha^{-1}$ ) decreases weakly with polymer concentration and is also (modestly) quantitatively sensitive to local chemical features such as the effective chain aspect ratio. ${ }^{44,59}$ Since $\left\langle|\mathrm{F}|^{2}\right\rangle / \rho_{\mathrm{m}} \propto\left(\mathrm{g}_{\mathrm{d}} \xi_{\rho}\right)^{2}$, this behavior reflects the fact that the density and chemical structure dependences of the interchain segmentsegment contact value and density sreening length are not precisely inversely proportional to each other with a universal prefactor. This conclusion seems unavoidable to some degree in any concentration regime where the physical requirement for scaling ideas to apply, $\xi_{\rho}$ $\gg \sigma$ or $\mathrm{d}$, does not hold. Thus, on the basis of these theoretical studies, we expect some (modest) chemical structure variability of $\alpha$ (or $\lambda_{\mathrm{D}}$ ) and a decrease (increase) in magnitude as the melt is diluted with solvent.

As discussed previously, 1,60 by comparing PMC theory with the reptation/tube theory in the asymptotic limit the parameter $\alpha$ is easily shown to be approximately 3 . Of course, this is only obviously relevant in polymer melts since it is the only case where the asymptotic $\mathrm{N}$-scaling of the diffusion coefficient is observed. In the reptation/tube picture, $\alpha$ is predicted to be universal, independent of chemical structure, polymer concentration, and solvent quality. Such universal ity of $\alpha$ results in a predicted concentration, solvent quality, and chemical structure independence of the constraint release corrections for viscosity and terminal relaxation time. Although not literally true for PMC/PRISM theory, these reptation/tube predictions of a relative insensitivity of $\alpha$ seem in rough accord with our microscopic approach. We note that computer simulations have never attained the asymptotic regime, so they provide no knowledge of the parameter $\alpha$.

We mentioned some potential limitations of the equilibrium structural model employed in paper I. The simple Ornstein-Zernicke form adopted for $S_{k}$ (see eq 1.45) is adequate for semidilute and moderately concentrated solutions. However, as the melt state is approached, the density screening length becomes so short that it is not obvious if such a form applies. However, the simple equilibrium model still seems justified since experimentally the inequalities $R_{g}, b \gg$ $\xi_{\rho}$ apply to entangled systems, ${ }^{49,56,58}$ and hence we only need to know $S_{k}$ for $k \xi_{\rho} \ll 1$. Another caveat is that we are treating a two-component polymer-solvent mixture as an effective one-component system. Although this is standard practice in coarse-grained polymer theories, it may be inadequate for accurately computing the chemical structure, temperature, and concentration dependence of the structural properties required by our microscopic dynamical theory. This fact, along with our neglect of hydrodynamic interactions, makes quantitatively-reliable a priori theoretical estimates of the $\alpha$ parameter especially difficult in solution.

Finally, we point out that both $\alpha$ and $\delta$ will, in general, depend (presumably weakly) on temperature via the implicit dependences of the relevant length scales, density, and equilibrium mean square forces. 


\section{Comparison with Melt and Solution Experiments}

A. Experimental Parameters. In order to test the description of finite size effects within the PMC theory, a number of experimental data sets are studied. The shear viscosities of melts of polybutadiene ${ }^{61}$ (PBD) and hydrogenated polybutadiene ${ }^{62}$ (PBDh) and the melt selfdiffusion coefficients of 63 PBD, PBDh, 62 polyisoprene ${ }^{63}$ (PI), poly(dimethylsiloxane) (PDMS), poly(ethylene oxide) $^{64}$ (PEO), and polystyrene ${ }^{65}$ (PS) are considered. In order to study the importance of the constraint rel ease mechanism in melts, measurements of PS tracers in PS matrices of different molecular weights ${ }^{66}$ and relaxation times from dielectric spectroscopy of PI tracers in PBD melts, ${ }^{9}$ and the neat PI melt case, are analyzed as well. Self-diffusion, $N=P$, and infinite-matrix-molecularweight-tracer diffusion coefficients, $\mathrm{P} \gg \mathrm{N}$, for three different concentrations of polystyrene dissolved in dibutyl phthalate, a good solvent, are also included. ${ }^{26-28}$ Here and throughout the paper, $\mathrm{P}$ denotes the molecular weight or degree of polymerization of the matrix polymers, and $\mathrm{M}$ or $\mathrm{N}$ the tracer values, respectively. Measurements extending to high reduced degrees of polymerization, $n=N / N_{e}$, are of special interest. The measurements of Nemoto and co-workers on semidilute and concentrated solutions of polystyrene are also included. At present, no convincing theoretical understanding of these data exists within the reptation/tube approach. ${ }^{29}$

Material parameters relevant to entangled polymer dynamics are listed in the table. The values of the molecular weights of entanglement, $\mathrm{M}_{\mathrm{e}}$, are the ones reported in the quoted experimental studies and used in our analysis. From the neutron scattering and rheological data survey of ref 46 , values for the entanglement length, $b$, and for the so-called packing length $p$ (and estimated density screening length $\xi_{\rho}$ ) are included. Within Gaussian thread PRISM theory, $\xi_{\rho}$ is rigorously identified with the packing length, $\mathrm{p}$. The data sets were shifted vertically on a logarithmic scale in order to determine the prefactors $\mathrm{k}_{\mathrm{B}} \mathrm{T} / \zeta_{0} \mathrm{~N}_{\mathrm{e}}$ or $\eta^{\mathrm{R}}$ that lead to overlap with the Rouse result, $D \xi_{0} N_{e} / k_{B} T \rightarrow 1 / n$ and $\eta / \eta^{\mathrm{R}} \rightarrow 1$, respectively, for small molecular weights, $\mathrm{n}=\mathrm{N} / \mathrm{N}_{\mathrm{e}}=\mathrm{M} / \mathrm{M}_{\mathrm{e}} \ll 1$. Note that in all cases data for $\mathrm{n} \leq 1$ were available, and as little arbitrariness was introduced in the shifts as possible. The PMC parameters, $\alpha$ and $\delta$, listed in the table follow from the fits discussed in the following sections and carry significant uncertainties.

B. Melt Viscosity. A PMC analysis of the reduced viscosity, $\eta / \eta^{\mathrm{R}}$, versus reduced molecular weight, $\mathrm{n}=$ $\mathrm{N} / \mathrm{N}_{\mathrm{e}}$, highlights the effects of the self-consistent constraint rel ease mechanism and involves one fit parameter only, the inverse strength parameter $\alpha$. Comparing the asymptotic reptation and PMC predictions for the viscosity at $n \rightarrow \infty$, the universal value $\alpha \approx 3.2$ is estimated. Figure 1 shows the viscosities of PBD melts for which the highest experimental reduced molecular weights have been reported. ${ }^{61}$ The theoretical result, eq 1.74, has been used. The PMC result (at least) semiquantitatively accounts for the measured data for all molecular weights. The asymptotic scaling, $\eta \sim \mathrm{N}^{3}$ (not shown in Figure 1), is not yet exhibited by the data nor by the PMC fit even though values $\mathrm{n}=10^{4}$ are achieved. The matrix constraints decay on the same time scale that determines the viscosity, i.e., the disentanglement time, $\tau_{\mathrm{D}}$, and less friction therefore arises than in the asymptotic limit of frozen entanglement

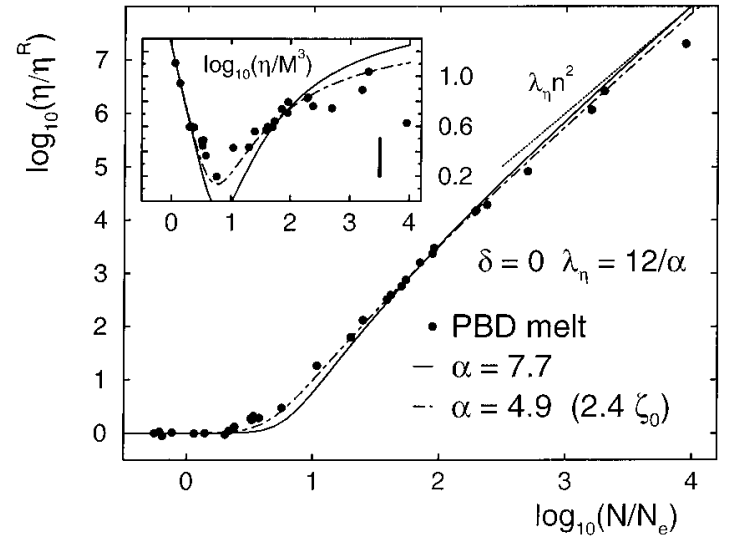

Figure 1. Reduced viscosity, $\eta / \eta^{\mathrm{R}}$, for a melt of polybutadiene $^{61}$ (PBD). The solid line is a one-parameter PMC fit using $\alpha=7.7$, the chain curve corresponds to $\alpha=4.9$, including the correction for the monomeric friction coefficient, $\zeta_{0}^{\mathrm{CFG}}=$ $2.364 \zeta_{0}$, suggested in ref 5 . The thin solid line is the asymptote for the second fit. The inset shows $\log _{10} \eta / \mathrm{M}^{3}$ shifted vertically by a constant (10) versus the logarithm of reduced molecular weight. The lines correspond to the fits in the main figure. A vertical bar denotes the error bar reported in ref 61 arising from uncertainties in the molecular weight and viscosity measurements.

constraints. The constraint porosity effects, i.e., the spatial correlations of the entanglements characterized by nonzero values of the density screening length, $\xi_{\rho}$, and the entanglement length, $b$, have been neglected in Figure 1, as they do not noticeably affect the shape of the theoretical curve for expected values of the length scale ratio in the melt, $\delta=\xi_{\rho} / \mathrm{b} \approx 1 / 20$.

A second fit of the PMC theory to the data is shown in Figure 1. It assumes, as has been pointed out by $\mathrm{O}^{\prime}$ Connor and Ball, 5 that the monomeric friction coefficient, $\xi_{0}$, found in the Rouse regime in ref 61 is somewhat too high. O'Connor and Ball argue by comparing the friction coefficients from ref 61 with an earlier measurement by Roovers, ${ }^{67}$ that the Rouse results of ref 61 are a factor 2.346 too high, $\xi_{0}^{\mathrm{CFG}}=$ $2.346 \xi_{0}$. If this correction of the monomeric friction coefficient is taken into account, by fitting the expression $\eta / \eta^{\mathrm{R}}=1+\left(\lambda_{\eta} / 2.364\right) \beta \mathrm{n}^{2}$ to the data instead of eq 1.74 , then a quantitative fit of the PMC results to the measured viscosities becomes possible in the full range of molecular weights excluding the highest data point only. The value found for the strength parameter $\alpha, \alpha$ $\approx 7.7$ without and $\alpha \approx 4.9$ with this correction, then lies closer to the reptation prediction of $\alpha \approx 3$ and also to values found in other polymer melts, e.g., PBDh (see below). Such values of $\alpha$ are also expected from the observation of the apparent power $\eta \sim \mathrm{N}^{3.4}$, as demonstrated in the model calculations shown in section 5.A of paper I, where a power $\eta \sim \mathrm{N}^{3.4}$ is observed for values of $\alpha$ below 5 .

After correction of the monomeric friction coefficient, all data except for the highest molecular weight point (which is not reliable ${ }^{68}$ ) fall close to the PMC fit. This is especially apparent from the inset in Figure 1 where the relative variation of the viscosity compared to its asymptotic behavior, $\eta / \mathrm{M}^{3}$, is shown. The data scatter rather unsystematically around the fit with deviations well compatible with the error bars reported in ref 61 due to uncertainties in the measurements of the molecular weight and the viscosity. The slow approach of the constraint release corrections to the asymptote, eq I.69, explains why the asymptotic $\eta / \mathrm{M}^{3} \rightarrow$ constant is not yet observed. 


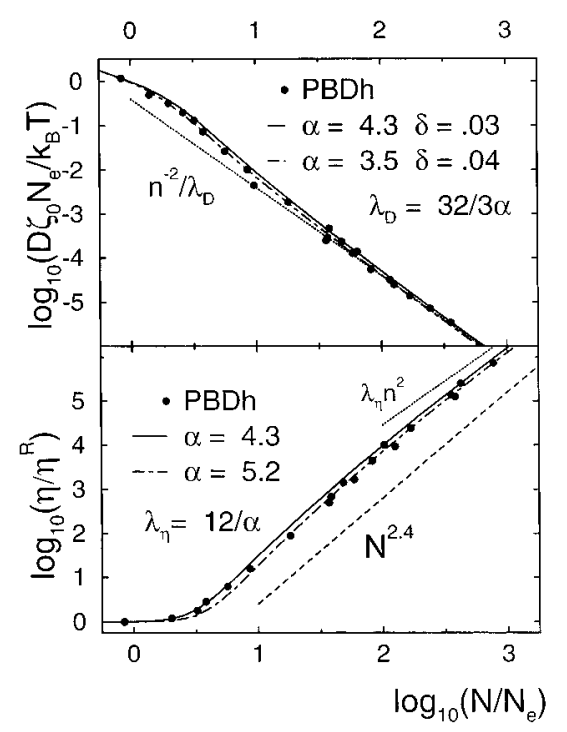

Figure 2. Self-diffusion constant (D, upper figure) and viscosity ( $\eta$, lower figure) versus reduced molecular weight for a melt of hydrogenated polybutadiene ${ }^{62}$ (PBDh). The solid lines correspond to fits to $\mathrm{D}$ and $\eta$ with a common parameter $\alpha=4.3$; additionally, $\delta=0.03$ is found for the diffusion constant. The thin solid lines are the asymptotic behaviors. The chain curves show the best independent fit leading to slightly different $\alpha$ (and $\delta$ for $D$ ). The viscosity data are compared to a power law $\mathrm{N}^{2.4}$ (short dashes).

From Figure 1, and especially from the inset, it is suggested that the apparent power law behavior of the viscosity in an intermediate molecular weight range, $\eta$ $\sim \mathrm{N}^{3.4}$, is not the sign of a true power law scaling behavior but arises from the competition of a finite size effect, namely the self-consistent constraint release mechanism, and the asymptotic scaling, $\eta \sim \mathrm{M}^{3}$. Clear observations of the true asymptotic power are not expected in the experimentally accessible molecular weight range, as the constraint release correction factor, $\beta\left(\mathrm{n} / \alpha^{2}\right)$, in eq I.67, shows an extremely slow approach to unity, $1-\beta \rightarrow 4 / 3\left(\alpha^{2} / n\right)^{1 / 4}$, eq I.73. Effectively, the slow variation of $\beta$ around $\mathrm{n} \approx 10^{3}$ may mimic the asymptote, eq I.55, which, however, correctly describes the data for much higher rescaled molecular weights $n$ only.

Unfortunately, no diffusion data exist for PBD melts out to such high molecular weights as used for the viscosity measurements. In the next section, the combined comparison of the theory with diffusion and viscosity data provides a further more stringent test of the physical picture of PMC theory.

C. Combined Viscosity and Self-Diffusion Melt Data. Of special value to test the close connection of the center-of-mass and conformational dynamics as is predicted by PMC theory are data on both dynamics for identical polymer systems. An interesting example are the measurements in PBDh by Pearson and co-workers, which extend to rather high degrees of polymerization and provide self-diffusion constants and viscosities for the identical samples. ${ }^{62}$ The viscosity analysis, using the PMC result, eq I.74, fixes the inverse strength parameter $\alpha$ and therefore the constraint release contribution to $\eta$ and $D_{\mathrm{s}}$. The value $\alpha=5.2$ gives the best least square fit to the viscosity data and is rather close to the value fitted to the PBD viscosities. Figure 2 displays the data and $\mathrm{PMC}$ results. A universal value of $\alpha$ is not expected by PMC theory; the magnitude agrees well with the PRISM estimate and roughly with the universal value predicted by reptation theory. The apparent power law behavior of the viscosity, ${ }^{62} \eta \sim \mathrm{N}^{3.4}$, is again explained as a finite size crossover effect due to the constraint release mechanism.

A slightly smaller parameter, $\alpha=4.3$, provides the combined best PMC fit to the viscosity and the selfdiffusion coefficient as a function of reduced molecular weight. The lower part of Figure 2 compares the two viscosity fits which exhibit similar fit quality. In the upper part of Figure 2 the self-diffusion coefficients are seen to overshoot slightly compared to the asymptote, $D \rightarrow D^{R} / \lambda_{D} n$, eq I.52. This small enhancement of the diffusion constant again results from the constraint release mechanism, as is shown by the PMC fit using eqs I.64, I.68, and I.69 and the same parameter, $\alpha=$ 4.3, as the viscosity fit. The small density screening length, $\delta=\xi_{\rho} / \mathrm{b} \approx 0.03$, found in this analysis indicates small constraint porosity contributions to D. Moreover, this value for $\delta$ is in good accord with the independently measured length scales listed in the table. An independent least squares fit to the diffusivities alone leads to a slightly smaller value of $\alpha=3.5$, but a somewhat larger constraint porosity contribution, $\delta=0.04$. The rather small variations of the parameters, $\alpha=4.3 \pm 1$ and $\delta=0.03 \pm 0.01$ for PBDh, however, are well compatible with both the simplifications of the polymer matrix model, eqs I.45 and I.46, used in the theoretical approach, and a priori estimates.

It is the decay of the matrix constaints that is the dominant finite size effect in polymeric melts. Therefore, the larger finite size corrections in the viscosity compared to the diffusion coefficients are expected in PMC theory. In paper I, it was discussed that the larger weighting of the amplitude of entanglement constraints for long wavelengths leads to larger constraint release effects in the conformational dynamics than in the center-of-mass motion. The observation in polymer melts of the asymptotic scaling, $\mathrm{D} \sim \mathrm{N}^{-2}$, in the selfdiffusion and tracer (for $\mathrm{P}>\mathrm{N}$ ) diffusion coefficients, but not in the shear viscosities, is therefore naturally explained by the different intramolecular correlations captured in the $\Sigma$ - and M-memory functions of PMC theory. See sections 4 and 5, and figures 4-8, in paper I for more discussions of this connection.

D. Melt Tracer and Self-Diffusion Constants. The small corrections of the finite size effects in the melt diffusion data have two origins in PMC theory. First, the constraint release mechanism is rather ineffective for the center-of-mass motion because of the local nature of the $\Sigma$-memory function. Second, in melts, the spatial correlations of the matrix constraints are characterized by rather small length scales, $\xi_{\rho}$ and b, and a length scale ratio $\delta=\xi_{\rho} / \mathrm{b}$, much smaller than in the solution cases.

Self-diffusion data for different melts do not fall on a single master curve, $D / D^{R}=f\left(N / N_{e}\right)$, as predicted by reptation theory, because of the variations in the (inverse) entanglement strength factor, $\alpha$. Independent fits to a number of polymer melt self-diffusion constants (PBD, PBDh, PDMS, PEO, PI, PS), however, show that values of $\alpha$ fall in a rather tight range of $\alpha=2.5-5$. The density screening lengths, $\xi_{\rho}$, and length scale ratios, $\delta=\xi_{\rho} / \mathrm{b}$, found in these fits lie consistently bel ow $\delta=0.06$, as expected for dense melts (see the Table). Figure 3 displays a representative set of self-diffusion data and corresponding PMC fits.

A particularly interesting set of self-diffusion data is included in Figure 3. Diffusion constants of PDMS ${ }^{64}$ 


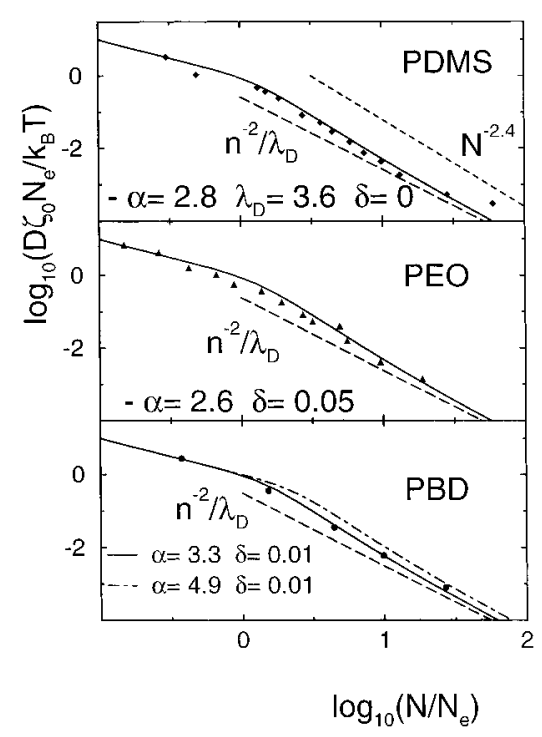

Figure 3. Self-diffusion constants in melts of poly(dimethylsiloxane) (PDMS), poly(ethylene oxide) ${ }^{64}$ (PEO), and polybutadiene ${ }^{63}$ (PBD) versus reduced molecular weight. PMC fits to the individual data sets are shown using the denoted parameters. For PEO and PBD, the relation $\lambda_{D}=32 / 3 \alpha$, eq 1.53 , is enforced. For PDMS, an unconstrained fit, varying $\alpha$, $\lambda_{\mathrm{D}}$, and $\delta$ is shown as solid line, and results in a small deviation of $\lambda_{\mathrm{D}}$ from its theoretical value, $32 / 3 \alpha \doteq 3.8$. A power law $\mathrm{D}$ $\sim \mathrm{M}^{-2.4}$ (short dashes) is compared to the PDMS data. The high molecular weight asymptotes are denoted by long dashed lines. The dot-dashed curve is a fit to the PBD data with the best $\alpha=4.9$ from the viscosity analysis of Figure 1 .

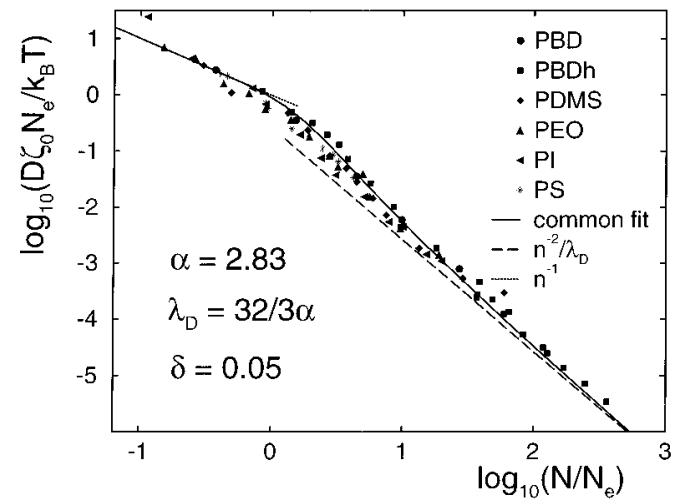

Figure 4. Self-diffusion constants for polymer melts versus reduced molecular weight. A common fit for the data of different polymers with the indicated parameters is shown as a solid line. The asymptotes, Rouse and reptation-like, are shown with prefactors corresponding to the fit.

exhibit an apparent power law, $\mathrm{D} \sim \mathrm{N}^{-2.4}$, up to ca. log $\left(\mathrm{N} / \mathrm{N}_{\mathrm{e}}\right)=1.2$. Such high apparent power law exponents for rather small degrees of polymerization are well rationalized by the PMC finite size effects, mainly the constraint release mechanism, as shown by the PMC fit in Figure 3. During this fit three parameters, $\alpha, \lambda_{D}$, and $\delta$, were varied freely in eqs I.64, I.68, and I.69. Supporting the close connection of the center-of-mass and the conformational motion predicted by PMC theory and leading to eq 1.53 for $\lambda_{\mathrm{D}}$, the two resulting fit parameters, $\alpha$ and $\lambda_{\mathrm{D}}$, almost satisfy eq I.53, 32/3 $\alpha \approx$ 3.8 compared to $\lambda_{\mathrm{D}}=3.6$.

A common PMC fit to a number of self-diffusion data sets using eqs I.64, I.68, and I.69 is shown in Figure 4. Rather small enhancements of the self-diffusion constants compared to the asymptotic value result from the small consequences of the constraint release mechanism in the melt. The small value of the extracted $\delta, \delta=$
0.05 , indicates that constraint porosity can be neglected in this fit.

Without showing a corresponding figure, let us report that for tracer polymers in a strongly entangled polymer melt, where the matrix molecular weight far exceeds the tracer molecular weight, $\mathrm{P} \gg \mathrm{N}$, almost no deviations from the asymptotic prediction, $D=D^{R} /\left(1+\lambda_{D} n\right)$, eq I.52, are observed in the PMC results, in agreement with experimental measurements. ${ }^{66}$ In Figure 7 of paper I, model calculations for melt-like parameters, $\alpha$ $=3$ and $\delta \leq 0.05$, show a smooth crossover of this infinite matrix molecular weight tracer diffusion constant from its Rouse, $\mathrm{D} \sim \mathrm{N}^{-1}$, to asymptotic, $\mathrm{D} \sim \mathrm{N}^{-2}$, behavior with no overshooting or higher apparent exponents in intermediate $\mathrm{N}$ regimes. This behavior clearly contrasts with the rather strong overshooting or rather high apparent exponent, $\mathrm{D} \sim \mathrm{N}^{-2.4}$, caused by the constraint release mechanism in the PDMS selfdiffusion data of Figure 3. Note that for $n=N / \mathrm{N}_{\mathrm{e}}=$ 4.4, the PDMS self-diffusion constant lies a factor 2.1 above the asymptote obtained by neglecting finite size effects. The individual analysis of the PBD and PEO self-diffusion coefficients in Figure 3 further support these findings; small length scale ratios are observed as well as dominating constraint release corrections to the asymptotic behavior.

The importance of the dynamics of the matrix constraints on the tracer diffusion coefficients can most comprehensively be studied from a set of data for different tracer, $M$, and matrix polymer, $P$, molecular weights. Recalling that our approach applies to entangled polymer matrices only, due to the approximations in the RR model, we study the data of Green and Kramer ${ }^{66}$ for PS tracer in PS melts for $\mathrm{P}>\mathrm{Pe}$. As the authors did not publish tables of their extended set of data, we use the reported fitted theoretical curves in order to compare with the PMC results. Note that Green and Kramer could fit their data equally well with the tube models of Graessley ${ }^{12}$ and Klein. ${ }^{14}$ Both models mainly differ in the steepness of the apparent $\mathrm{P}$ dependence of the tracer diffusion constants in the crossover range, $\mathrm{P} \leq \mathrm{N}$. We use the fitted model results of Graessley because we expect easier agreement of PMC theory with the model by Klein; in Figures 8 and 9 of paper I, P dependences around Klein's prediction, $\mathrm{D} \sim \mathrm{P}^{-2.5}$, are seen in the PMC results. Graessley's expression exhibits a stronger $\mathrm{P}$ dependence,

$$
D(M, P) \propto \frac{1}{M^{2}}\left(1+\alpha_{c r} M_{e}^{2} M P^{-3}\right)
$$

Note that eq 1 does not reproduce the Rouse model for small tracer molecular weights, $\mathrm{M}<\mathrm{M}_{\mathrm{e}}$. Therefore, we normalize it to unity for $M=M_{e}$, i.e., study $D(M, P) /$ $D\left(M_{e}, P\right)$, and do not require the connection of $\alpha$ and $\lambda_{D}$, eq I.53, i.e., the relative ratio of Rouse to asymptotic PMC result, in the PMC fit. The experimental parameters, $M_{e}$ as in the table and $\alpha_{c r}=(48 / 25) z\left(12 / \pi^{2}\right)^{z-1}$ with $z=3.5$, are taken from ref 66 and therefore mimic the experimental data for the PS tracer in PS melt systems. The values for the tracer and matrix polymer molecular weights used in Figure 5 span the experimentally studied range. Even though the $\mathrm{PMC}$ results in Figure 5 do not reproduce the $D \sim P^{-3}$ prediction of Graessley for the tracer diffusion constant in the constraint release dominated region, overall a reasonable fit to Graessl ey's model can be achieved as shown. The value $\alpha=3$ is chosen a priori as appropriate for polymer melts, and the parameters $\delta=0.07$ and $\lambda_{\mathrm{D}}=1.58$ are found from 


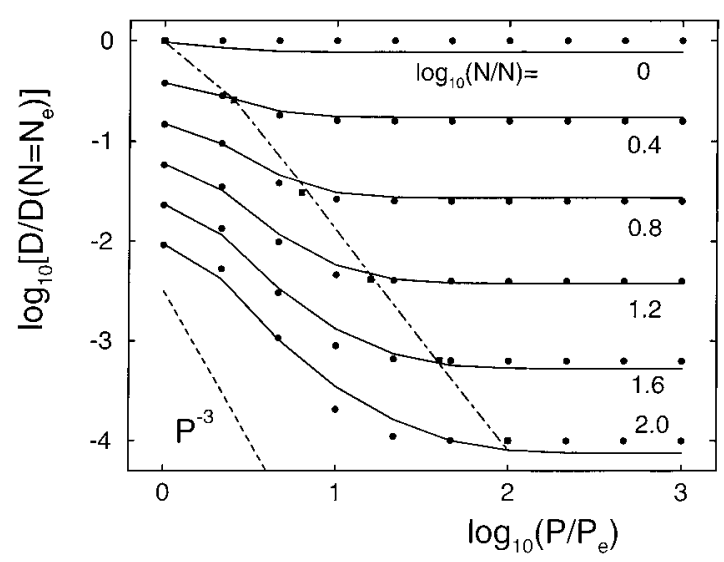

Figure 5. Tracer diffusion coefficients versus matrix molecular weight, $p=P / P_{e}$, for different tracer molecular weights, $\mathrm{n}=\mathrm{N} / \mathrm{N}_{\mathrm{e}}$, as specified in the figure. The model of Graessley, ${ }^{12}$ eq 1, which has been fitted to polystyrene (PS) melts by Green and $\mathrm{K}$ ramer, ${ }^{66}$ is shown by circles for $n \neq p$ and by squares for $\mathrm{n}=\mathrm{p}$. The PMC fit is shown by the solid curves; parameters are $\alpha=3$ (a priori fixed), $\delta=0.07$, and $\lambda_{\mathrm{D}}=1.58$. The corresponding self-diffusion coefficients are indicated by a chain curve.

least squares fits. When the shapes of the PMC results are compared with the actual diffusion data of ref 66 , qualitative agreement can be deduced. It appears that the PMC theory adequately describes the constraint release decay of the matrix entanglement constraints, as observed in tracer diffusion measurements in entangled polymeric melts.

E. Dielectric Relaxation Times. An especially powerful technique to study the conformational tracer dynamics, especially the end-to-end vector fluctuations, is the dielectric spectroscopy of type-A tracer polymers in type-B polymer melts.9,10 Type-A polymers are defined to possess monomeric dipole moments parallel to the chain contour, whereas type-B polymers possess perpendicular moments only. ${ }^{69}$ Adachi and co-workers have studied such a system, PI tracer in PBD melts, for varying tracer and matrix molecular weights. ${ }^{9}$ They report the molecular weight dependence of the tracer dielectric relaxation time and the high-frequency asymptote of the disentanglement process in the dielectric spectrum. It is important to recall from paper I that PMC theory identifies two different mechanisms, one which is responsible for the initial decay of the end-toend vector correlation function, $\langle\mathbf{P}(\mathrm{t}) \cdot \mathbf{P}(0)\rangle\langle\langle\mathrm{P}(0) \cdot \mathbf{P}(0)\rangle-$ $1 \sim\left(\mathrm{t} / \mathrm{N}^{\mathrm{y}} \tau_{0}\right)^{\mathrm{x}}$, and two other ones which affect the dielectric relaxation time. At first, let us discuss the latter, namely the constraint release and constraint porosity effects, and compare them with the experimental data for $\tau^{\epsilon}$, the dielectric relaxation time.

As shown in section 5.D of paper I, PMC theory predicts three different asymptotic behaviors for the relaxation time of the end-to-end vector correlation function of a tracer polymer. In unentangled matrices, $\mathrm{p}=\mathrm{P} / \mathrm{P}_{\mathrm{e}}<1$, the Rouse behavior, $\tau^{\epsilon} \sim \mathrm{N}^{2}$, should be observed. In strongly entangled matrices, where the surrounding polymers are immobile relative to the tracer, the dielectric follows the internal disentanglement time, $\tau^{\epsilon} \propto \tau_{\mathrm{D}}$, and exhibits reptation-like scaling, $\tau^{\epsilon} \sim N^{3}$. For tracers which are much larger than the matrix polymers, which are entangled, however, i.e., $\mathrm{n}$ $=\mathrm{N} / \mathrm{N}_{\mathrm{e}} \gg \mathrm{p} \gg 1, \mathrm{PMC}$ theory finds an intermediate scaling behavior, $\tau^{\epsilon} \sim \mathrm{N}_{\mathrm{e}}^{2} \mathrm{p}^{3 / 4} n^{19 / 8}$. Note that these results, more strongly than our results for the internal or the center-of-mass dynamics, depend on the technical approximations made when diagonalizing the equations

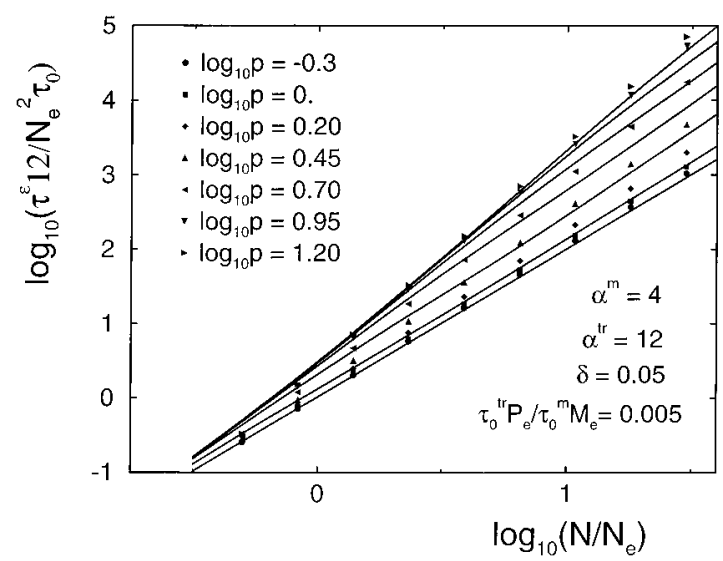

Figure 6. Dielectric relaxation times, $\tau^{\epsilon}$, according to eq 2 , of a polyisoprene tracer ${ }^{9}$ versus reduced tracer molecular weight, $\mathrm{N} / \mathrm{N}_{\mathrm{e}}$, for the indicated various polybutadiene matrix molecular weights, $p=P / P_{e}$. The lines correspond to $P M C$ results from eq I.70 with the parameters specified in the figure and the table.

of motion, eq I.1, where chain end effects have been neglected. 60

Also, for a PI tracer in a PBD matrix, system-specific polymer interactions could strongly influence these results, especially the parameters characterizing the tracer dynamics. Whereas the parameters, $P_{e}, \alpha^{m}$, and $\delta$, specifying the dynamics of the entangled melt correspond to the ones found (for example) from independent viscosity measurements, the tracer parameters, $\mathrm{N}_{\mathrm{e}}$ and $\alpha^{\text {tr }}$, must differ from the corresponding values found for the one-component system of tracer polymers. As eqs I.35 and I.54 show, these parameters are determined by the tracer-matrix interactions. Moreover, the importance of the constraint release mechanism depends on the ratio of the monomeric time scales of the tracer and matrix polymers. The corresponding variable is $r=\tau_{0}^{\mathrm{tr}} \mathrm{P} d \tau_{0}^{\mathrm{m}} \mathrm{N}_{\mathrm{e}}$ in eq I.64, where $\tau_{0}^{\mathrm{tr}}$ is the monomeric time scale of the tracer in the specific matrix it is embedded in, not in its own melt state.

Adachi and co-workers have observed that the dielectric relaxation times, $\tau^{\epsilon}$, of PI tracers in PBD matrices measured in the molecular weight ranges, $-0.2 \leq \log _{10}$ $\mathrm{n} \leq 1.45$ and $0.0 \leq \log _{10} \mathrm{p} \leq 2.03$, can be fitted with the following formula:

$$
\begin{aligned}
\log _{10}\left(\tau^{\mathrm{e}} / \mathrm{N}_{\mathrm{e}}{ }^{2} \tau_{0}\right) & = \\
& -1.92+\left(3-\mathrm{e}^{-(\mathrm{P} / 8500)^{1.5}}\right) \log _{10}(\mathrm{M} / 1900)
\end{aligned}
$$

where $\tau_{0} \mathrm{Ne}^{2}=10^{-3.71} \mathrm{~s}$ and the molecular weights of entanglement are $\mathrm{P}_{\mathrm{e}}=1850$ and $\mathrm{M}_{\mathrm{e}}=5000$. Note that the range $0.03<\log _{10} p<1.03$ is of special interest, as there the effective exponent is found to lie between the expected asymptotic behaviors, $\tau^{\epsilon} \sim \mathrm{N}^{2}$ or $\tau^{\epsilon} \sim \mathrm{N}^{3}$. These limits, Rouse for $\mathrm{P}<\mathrm{P}_{\mathrm{e}}$ and reptation-like for $\mathrm{P}$ $\gg \mathrm{P}_{\mathrm{e}}$, are also predicted by $\mathrm{PMC}$ theory. For intermediate matrix molecular weights, the PMC approach does not predict simple power laws as reported ${ }^{9}$ in eq 2 . A crossover from $\tau^{\epsilon} \sim \mathrm{N}^{3}$ for $\mathrm{N} \ll \mathrm{P}$, to $\tau^{\epsilon} \sim \mathrm{N}^{19 / 8}$ for $\mathrm{N} \gg$ $P$, for entangled matrices, $P>P_{e}$, results in some curvature. The PMC curves matched to eq 2 therefore do not fit quantitatively. In the comparison of Figure 6 the PBD melt is a priori characterized by parameters, $\alpha=4$ and $\delta=0.05$, found previously in sections 3.A and 3.B. Then three parameters are varied in the fitting procedure. For simplicity, $\mathrm{M}_{\mathrm{e}}$ of the $\mathrm{PI}$ tracer in the PBD melt is chosen to be the one for a PI melt, and $\alpha^{\text {tr }}$ 


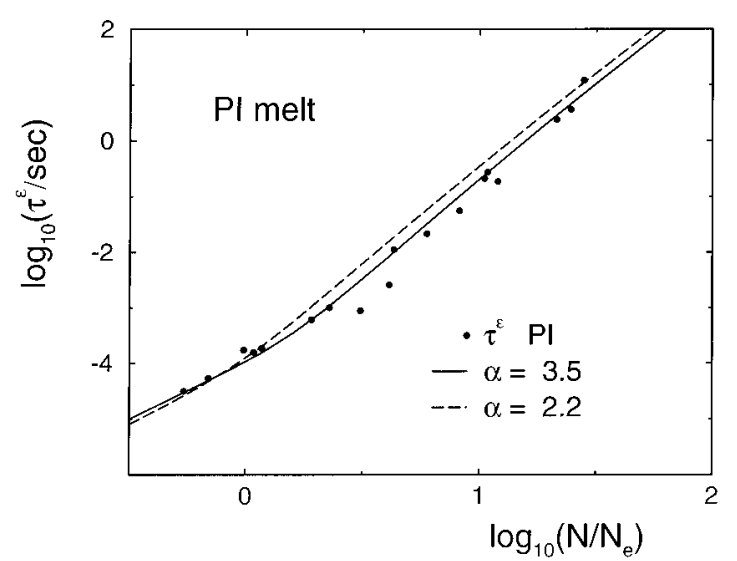

Figure 7. Dielectric relaxation times for polyisoprene (PI) melts ${ }^{24}$ versus reduced molecular weight. The PMC curves are calculated for the parameters $\alpha=3.5$ (solid line) and $\alpha=$ 2.2 (dashed line), where the latter value is taken from the fit to the PI self-diffusion constants shown in Figure 4 and exhibits large uncertainties; the a priori chosen melt-like value $\delta=0.05$ is used.

$=12$ is matched to the asymptotic behavior, $\tau^{\epsilon} / \mathrm{Ne}_{\mathrm{e}}^{2} \tau_{0} \sim$ $(32 / 12)\left(n^{3} / \alpha^{\text {tr }}\right)$. The fitted small value of the time scale ratio, $r=\tau_{0}^{\text {tr }} \mathrm{P}_{e} \tau_{0}^{\mathrm{m}} \mathrm{N}_{\mathrm{e}}=0.005$, indicates that the tracer polymer is relatively mobile compared to the matrix polymers. As is evident from eq I.64, the effect of a small parameter $r$ can also be interpreted as a change in $\mathrm{N}_{\mathrm{e}}, \Theta \propto \mathrm{rN}_{\mathrm{e}}^{-5 / 2}$, and without further independent experimental information a closer identification of the PMC model parameters is impossible. Also, the errors introduced into the $\mathrm{PMC}$ results by the neglect of chain end corrections ${ }^{60}$ are unknown at present. In Figure 6 , the reported change ${ }^{9,10}$ from Rouse to reptation-like molecular weight scaling of $\tau^{\epsilon}$ is qualitatively correctly reproduced by the PMC results, but with quantitative deviations. Instead of a scaling exponent that continuously depends on matrix molecular weight, ${ }^{9}$ three exponents, 2, 19/8, and 3, are predicted, whose observation depends on the ratios N/P and $\mathrm{P} / \mathrm{P}_{\mathrm{e}}$.

Let us recall the predicted behavior of the melt dielectric relaxation time shown in Figure 10 of paper I. There, the dielectric relaxation time of one of the matrix polymers, i.e., $\mathrm{N}=\mathrm{P}$ and identical chemistry, was observed to scale roughly like $\tau^{\epsilon} \sim \mathrm{N}^{3.4}$ in an intermediate molecular weight range, $0.5 \leq \log _{10}(\mathrm{~N} /$ $\mathrm{N}_{\mathrm{e}}$ ) $\leq 2$. Figure 7 shows the dielectric relaxation times for PI homopolymer melts $22-24$ and PMC results for melt like parameters $\alpha=2.2-3.5$ and $\delta=0.05$. Both values agree with the estimates given in section 2 and are compatible with the analysis of the self-diffusion data ${ }^{63}$ shown in Figure 4. Thus, PMC theory also achieves a quantitative description of the PI melt dielectric relaxation times mainly by adjusting the self-consistent constraint release mechanism effects via the parameter $\alpha$.

The molecular weight dependence of the dielectric relaxation time thus is influenced by the finite size effects that result from the spatial and temporal correlations of the matrix constraints. The initial decay of the disentanglement process in the end-to-end vector correlation function, however, is determined by the tracer shape fluctuations in PMC theory. As shown in section 3.A of paper I, the reptation-like behavior, $\epsilon^{\prime \prime}\left(\omega \tau^{\epsilon} \gg 1\right) \sim \omega^{-1 / 2}$, emerges from the PMC description only if the internal contributions to the collective tracer dynamics in the RR model are neglected, which is not

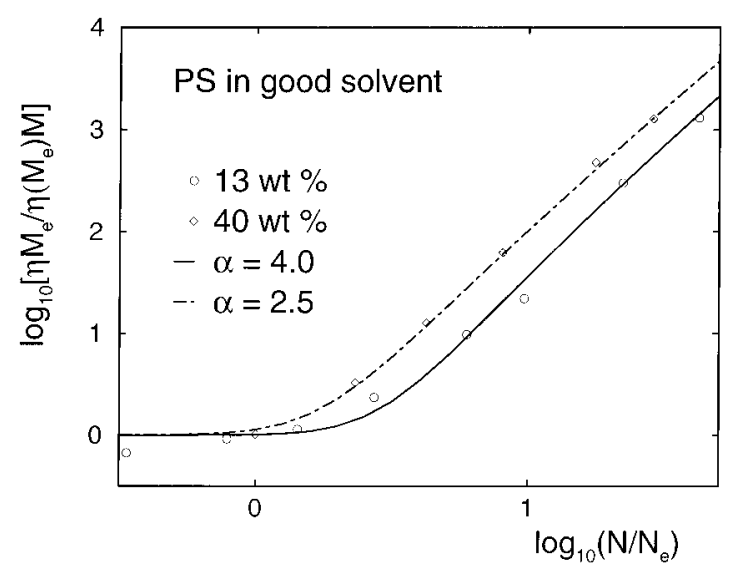

Figure 8. Viscosities of solutions of 13 wt \% (circles) and 40 wt \% (diamonds) polystyrene in a good solvent. 26,28 The PMC fits use the parameters $\alpha=4.0$ (solid line) for the 13 wt \% and $\alpha=2.5$ (chain curve) for the $40 \mathrm{wt} \%$ data.

justifiable. Even in the case of tracer motion in matrices of immobile polymers, where the asymptotic scaling, $\tau^{\epsilon}$ $\sim \mathrm{N}^{3}$, is predicted, the tracer shape fluctuations of PMC theory still speed up the initial disentanglement process, resulting in $\epsilon^{\prime \prime}\left(\omega \tau^{\epsilon} \gg 1\right) \sim \omega^{-x}$, with 9/32 $\leq x \leq 3 / 8$ (see section 3.A of paper I). These predictions qualitatively agree with the observation ${ }^{9}$ of (i) frequency power law exponents around $\mathrm{x}=0.22-0.31$ for $\mathrm{Pl}$ tracers in strongly entangled $\mathrm{PBD}$ matrices $\left(\mathrm{N} \ll \mathrm{P}\right.$ and $\mathrm{P} \gg \mathrm{Pe}_{\mathrm{e}}$ ), where $\tau^{\epsilon} \sim N^{3}$, (ii) exponents $x \approx 0.53$ in the Rouse limit, $\mathrm{P}_{\mathrm{e}} \ll \mathrm{N} \ll \mathrm{P}$, and (iii) $\mathrm{x}$ values in between for matrix molecular weights in between. Only numerical calculations ${ }^{70,71}$ based on the full PMC equations can determine whether quantitative agreement results.

F. Solution Transport Coefficients. It has been rather well established that the molecular weight dependence of the viscosity of entangled polymeric solutions is very similar, and perhaps identical to within experimental error, to the behavior in polymer melts.50,72 For example, in solutions of PS in a good solvent, Nemoto and co-workers ${ }^{26,28}$ found that for the semidilute and concentrated solutions the viscosity shows an apparent power law behavior, $\eta \sim \mathrm{N}^{3.5 \pm 0.1}$ in the range $0 \leq \log \left(\mathrm{N} / \mathrm{N}_{\mathrm{e}}\right) \leq 1.7$. As Figure 8 shows, $\mathrm{PMC}$ theory can quantitatively account for these behaviors, and (inverse) entanglement strength factors in the physically expected range of $\alpha=2.5$ for 40 wt \% PS and $\alpha=4.0$ for 13 wt \% PS are found. The similarity of the entangled solution viscosity to the melt viscosities finds a clear physical origin within PMC theory in the similarity of $\alpha$ for solutions and melts. From the PRISM estimates, this density and concentration independence of $\alpha$ has been anticipated. For the semidilute concentration data, 13 wt \% in Figure 8, it is not clear how to unambiguously compare theory and experimental data. For low molecular weights, $\mathrm{N} \leq \mathrm{N}_{\mathrm{e}}$, the data exhibit a slightly stronger dependence on molecular weight than explained by the Rouse model, which is assumed in the PMC approach to apply for $\mathrm{N}<\mathrm{N}_{\mathrm{e}}$. The vertical shift of the 13 wt \% data therefore is unclear. We estimate that this is the origin of the variation of $\alpha$ between 2.5 and 4 found in the fits of Figure 8; these fits result if the experimental viscosities are normalized to $\eta / M=1$ for $\mathrm{M}=\mathrm{M}_{\mathrm{e}}$

The PMC theory together with the PRISM estimates of the dynamical parameters explain that viscosity versus molecular weight curves for solutions and melts 


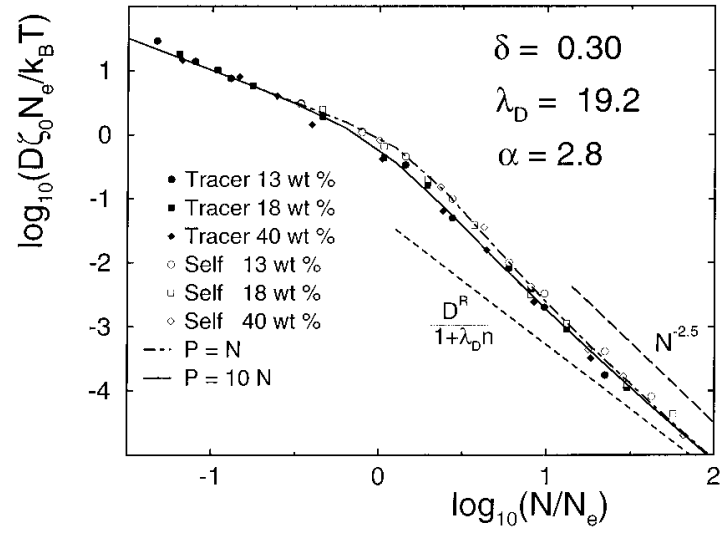

Figure 9. Self-diffusion (open symbols) and tracer diffusion (filled symbols) constants for solutions of polystyrene (PS) in dibutyl phthalate (good solvent). The reported power law, D $\sim \mathrm{N}^{-2.5}$, is indicated by a long dashed line. ${ }^{26-28} \mathrm{~A}$ common fit to the self-diffusion (chain curve, identical tracer, $N$, and matrix polymer, $\mathrm{P}$, degree of polymerization) and tracer diffusion (solid line, $\mathrm{P}=10 \mathrm{~N}$ ) data for the three concentrations is shown and differs in the constraint release contribution only; a strong overshoot compared to the asymptote without finite size effects, $D=D^{R} /\left(1+\lambda_{D} n\right)$ (short dashes), arises from the constraint porosity. The theoretical connection of $\alpha$ and $\lambda_{\mathrm{D}}$ is broken.

are similar. Note that the theoretical predictions for the concentration dependence of $\mathrm{N}_{\mathrm{e}}$ can in principle be used, although in our case $\mathrm{N}_{\mathrm{e}}$ was taken from experiment. ${ }^{26-28}$ The similarity of solution and melt viscosities results in PMC theory from the equal influence of the matrix constraint decay relative to the probe diffusive motion in the conformational memory function, $M(t)$. I mportant for this result are two physical aspects. First, rather long ranged intramolecular correlations dominate the conformational friction function; see the discussion of $\mathrm{I}_{\mathrm{q}}^{\mathrm{M}}$, eq I.63. This leads to insensitivity of $\eta$ to local length scal es like the density screening length. Second, the (near) cancelation of the concentration dependence in the PMC entangl ement strength factor, ${ }^{44}$ $1 / \alpha \sim \mathrm{gd}_{\mathrm{d}}{ }^{2} \mathrm{~S}_{0} \sim \rho_{\mathrm{m}}^{0}$. Although at lower solution densities the polymer density fluctuations, or the osmotic compressibility, are strongly enhanced (Iarger $\left.S_{0}\right)$, the local contact probabilities of monomers on different polymers, $g_{\text {d }}$, diminish, thereby resulting in $\alpha \approx$ constant with varying polymer concentration.

When the tracer and self-diffusion coefficients of the same PS in good solvent systems are studied, ${ }^{26-28}$ it is the constraint release mechanism that leads to the differences between the self, $N=P$, and (infinite matrix molecular weight) tracer, $\mathrm{P} \gg \mathrm{N}$, diffusion coefficients. In Figure 9 one observes a similar enhancement of the solution self-diffusion constants rel ative to the solution tracer diffusivities, as has been observed in Figure 3 for the melt diffusion data. There the melt self-diffusion constant at $\mathrm{N} / \mathrm{N}_{\mathrm{e}}=4.4$ was a factor 2.1 larger than the asymptotic expression, eq I .52, which neglects finite size and especially constraint release effects. Due to the similar constraint release parameter, $\alpha=2.8$, a priori chosen in the analysis of Figure 9, a similar enhancement of the self-diffusion data (affected by constraint release) to the $\mathrm{P} \gg \mathrm{N}$-tracer diffusion constants (unaffected by constraint release) is observed. F or example, at $\mathrm{n}=\mathrm{N} / \mathrm{N}_{\mathrm{e}}=4.4$ for the 40 wt $\%$ PS solution, $\mathrm{D}(\mathrm{N}=$ $\mathrm{P}) / \mathrm{D}(\mathrm{P} \gg \mathrm{N}) \approx 2.1$ is found in Figure 9 .

Even though the constraint release mechanism cannot be of relevance to the (infinite matrix molecular weight) tracer diffusion constants, they do not exhibit the reptation-like scaling, $\mathrm{D} \sim \mathrm{N}^{-2}$, in dense PS solutions. ${ }^{26-28}$ The experimental data of Nemoto and co-workers ${ }^{26-28}$ which extend to rather high molecular weights, $\mathrm{n} \leq 70$, exhibit an apparent power law behavior, $\mathrm{D} \sim \mathrm{N}^{-2.5 \pm 0.1}$, and lie well below the corresponding melt data at high molecular weights plotted in an isofriction comparison for fixed $n=N / N_{e}$. In Figure 9 the measured diffusion data are compared to this power law, to a PMC fit, and to the asymptotic PMC prediction, eq I.52 with $\lambda_{\mathrm{D}}=19.2$. The PMC fit uses an independently, from the viscosity analysis, determined $\alpha$, and freely varies $\delta$ and $\lambda_{\mathrm{D}}$. Note that the short dashed curve in Figure 9 shows the asymptotic $P M C$ result including the Rouse, unentangled behavior, i.e., $D=D^{R} /\left(1+\lambda_{D} n\right)$. The enhancement of the diffusion, especially of the tracer diffusion coefficients, relative to the asymptotic behavior, $\mathrm{D} \sim \mathrm{N}^{-2}$, therefore cannot result from the crossover to the Rouse law, $D \sim 1 / N$. The enhancement of the PS diffusion coefficients in entangled, PS solutions relative to the short dashed asymptote in Figure 9 is a finite size effect according to the PMC fits included in Figure 9.

From the true $P M C$ asymptote, $D \sim D^{R} / \lambda_{D} n$, one can deduce that the asymptotic prefactor, $\lambda_{\mathrm{D}}$, which, in the comparison with the melt diffusion constants in Figure 4 , was found to be around 3 in melts, has to be appreciably larger in solutions. A value of $\lambda_{D}$ smaller than $\lambda_{D}=12$ appears not compatible with the solution data, as they exhibit an increased molecular weight dependence out to the highest $\mathrm{N}$ data points. Such a large value of $\lambda_{D}$ is not compatible with the relation $\lambda_{D}$ $=32 / 3 \alpha$, eq I.53, as the value of $\alpha$ lies between 2 and 5 , as has been discussed in context with the solution viscosity and the difference of self-diffusion to tracer solution diffusion constants. At present it is not dear how this violation of eq I.53 can arise in PMC theory. One possible origin is the simplified treatment of the two-component system, i.e., polymer solvent mixture, as an effective one-component system. Lacking a quantitative theoretical understanding of $\lambda_{D}$ in solutions, we suggest using it as a free fit parameter in eqs I .64, I.68, and I.69, while $\alpha=3 \pm 1$ may be fixed a priori from independent viscosity measurements.

The PMC fits to the self-diffusion and tracer diffusion constants in entangled, concentrated polymer solutions in Figure 9 thus show that again the apparent power law behavior, $\mathrm{D} \sim \mathrm{N}^{-2.5}$, can be accounted for by a finite size correction of PMC theory, the constraint porosity. It arises from the rather large density screening length, $\delta=\xi_{\rho} / \mathrm{b}=0.3$, found in the fits. Note that the constraint porosity corrections, which cause the strong deviations of the solution diffusivities from the asymptote, $D \sim N^{-2}$, in Figure 9 have no analog in the reptation/tube picture. Consequently, no consistent theoretical understanding within that approach has up to now been presented for the solution data of Nemoto and co-workers, ${ }^{29}$ which explains the difference ${ }^{30,31}$ of solution and melt diffusivities and the melt results as well.

Within the PMC theory, the enhanced effective exponent results from the gradual buildup of the full constraint amplitudes a tracer polymer feels as it compresses the surrounding entanglement mesh. Only when the size of the polymer tracer far exceeds the entanglement length, $b$, and the compressibility or density screening length scale, $\xi_{\rho}$, are the full matrix constraint amplitudes effective and lead to the asymptotic behavior. As the reptation/tube approach neglects the spatial variation of the compressibility by simplify- 


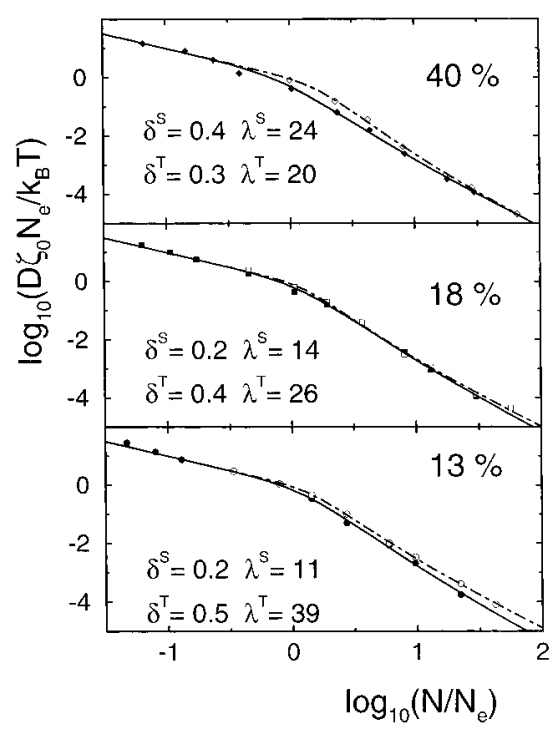

Figure 10. Independent PMC fits to the self-diffusion (S; open symbols) and tracer diffusion ( $T$; filled symbols) constants for solutions of polystyrene (PS) in dibutyl phthalate (good solvent) from refs 26-28; $\alpha=2.8$ is kept fixed for all fits, and the found parameters $\delta$ and $\lambda_{\mathrm{D}}$ are listed in the figure. Error bars for $\delta$, $\delta=0.33 \pm 0.12$, and $\lambda_{\mathrm{D}}=22 \pm 10$ follow.

ing the spatial correlations of the entanglement constraints to arise solely from the entanglement length $(\delta=0$ effectively), it misses this effect and cannot explain the solution diffusion coefficients. The PMC approach naturally includes the constraint porosity effects as it starts from the microscopic correlations of the polymeric liquid structure. This is especially necessary in polymer solutions where the density screening length is not small compared to the entanglement length; $\delta=\xi_{\rho} / \mathrm{b}=0.3$ is found for the PS solution data of Nemoto et al. ${ }^{26-28}$ This length scale ratio is larger in solutions than in melts, as has been argued in section 2. It is rather intriguing, but not understood at present, that the ratios of the asymptotic prefactor, $\lambda_{D}$, in solution to the one in melts and of the length scale $\delta$ in solution to its value in melts, are rather similar: $\lambda_{\mathrm{D}}^{\text {sol } /}$ $\lambda_{\mathrm{D}}^{\text {melt }} \approx \delta^{\text {sol }} / \delta^{\text {melt }} \approx 5-6$.

The possibility of a common fit with one set of parameters of self-diffusion relative to its tracer diffusion analog at three different polymer concentrations in good solvents shows that the constraint release enhancement of self-diffusion to tracer diffusion constants is correctly captured in PMC theory, and that the PRISM prediction of concentration independence of the parameters $\alpha$ and $\delta$ in solutions is accurate. Note that variations of $\alpha$ around $\alpha=3 \pm 1$ do not worsen the fits. This point is addressed in Figure 10 by independent least squares fits to the individual data sets; $\alpha=2.83$ is a priori fixed. Variations of $11 \leq \lambda_{D} \leq$ 39 and $0.2 \leq \delta \leq 0.5$ again emphasize that both parameters are larger for solutions than for melts but do not exhibit systematic polymer concentration dependences in the semidilute and concentrated solution regimes.

The enhancement of the diffusion coefficients compared to the asymptotic result, $\mathrm{D} \sim \mathrm{N}^{-2}$, is considerable even for large molecular weights. For $\mathrm{n}=\mathrm{N} / \mathrm{N}_{\mathrm{e}}=4.4$ and the 40 wt \% PS solution, the tracer diffusion coefficient lies at a factor 6 , and the self-diffusion constant at a factor 12 , above their common asymptote in Figure 9; at $\mathrm{n}=30$ for the $18 \mathrm{wt} \%$ solution the enhancement factors are still 2.2 and 1.9, respectively.
Extending the PMC fits, a merging of the diffusion data with the asymptote can be expected only for even larger molecular weights, ca. $\mathrm{n} \approx 300$.

\section{Polymer Tracer Motion in Gels: Comparison with Experiment}

As the extension of the PMC theory to polymer tracer diffusion through gels has been presented in paper I, and since tracer motion through gels is the original problem studied by de Gennes, ${ }^{73}$ two experimental studies of this problem are included in this paper. The low-field mobilities, $\mu=D N$, from electrophoresis measurements of poly(styrenesulfonate) (PSS) in polyacrylamide (PAA) gels, ${ }^{74}$ and the tracer diffusion measurements of PS in poly(vinyl methyl ether) (PVME) gels, ${ }^{75}$ are considered.

The PMC theory can also describe the motion of polymer tracers through random materials, where especially gels are of interest (see paper I). The physical aspects of constraint porosity in the PMC description naturally generalize to fractal media like gels. Two different gel models were mentioned: "hard gels", eq 1.76, which are characterized by rigid density structures that pose the constraints on the tracer dynamics, and "soft gels", eq I.77, where the flexibility of the matrix structure results in much smaller elastic constraints. Simple extensions of the PMC results for polymer melts and solutions just replace the tracer and matrix equilibrium structure by a fractal tracer or gel structure, respectively. Due to the time independence of the arrested gel structure, the constraint release mechanism is absent in gels or amorphous solids in general. In PMC theory strong enhancements of the tracer diffusivities relative to the asymptotic power laws are predicted from the fractal generalizations of the constraint porosity effects.

As the tracer-gel chemical interactions may show strong variations, the possibility to make a priori PRISM estimates for the dynamical parameters is missing at the present state of the art of PRISM..$^{51,52}$ From physical considerations, as the entanglement asymptotic prefactors, i.e., the $\lambda$ 's in eqs $1.67,1.68$, and 1.79, clearly are proportional to the tracer matrix interactions, $\alpha^{-1} \propto \lambda \propto\left\langle\left|\mathrm{F}_{\mathrm{tg}}\right|^{2}\right\rangle / \rho_{\mathrm{m}}$, where the $\boldsymbol{F}_{\mathrm{tg}}$ are the tracer-gel interaction forces, and as stronger chemical interactions, like preferential adhesion, clustering, or partial wetting, are expected for polymers in gel pores, it can safely be concluded that the asymptotic prefactor $\lambda_{\mathrm{g}}$ in eq 1.79 may be significantly larger than estimated for simple homopolymer solution or melt systems.

As has been shown in section 6 of paper I, the most important variable determining the tracer diffusivities is the ratio of gel pore size, $\xi_{g}$, to tracer size, $\mathrm{R}_{\mathrm{g}}$. Large finite size corrections are predicted by eqs I.79 and I.80 for tracers that persist even if $R_{g} \gg \xi_{g}$ but are not yet in the true asymptotic limit, which may require $\mathrm{R}_{\mathrm{g}} / \xi_{\mathrm{g}}>100$. As the asymptotic regime is reached when $\mathbf{R}_{\mathrm{g}} \gg \xi_{g}$, changes in the mass-size scaling of the tracer polymer do affect the shape of the preasymptotic apparent scaling region. Variations in the gel fractal dimension, however, mainly shift the fitted (unknown) material parameters but do not significantly influence the shape of the $\mathrm{D}(\mathrm{N})$ curves.

Figure 11 reproduces the low-field mobility data, ${ }^{74} \mu$ $=\mathrm{DN}$, of PSS in PAA gels of different gel concentrations, c. From the data in Figure 11 it is apparent that much stronger molecular weight dependences of $\mu$ are ob- 


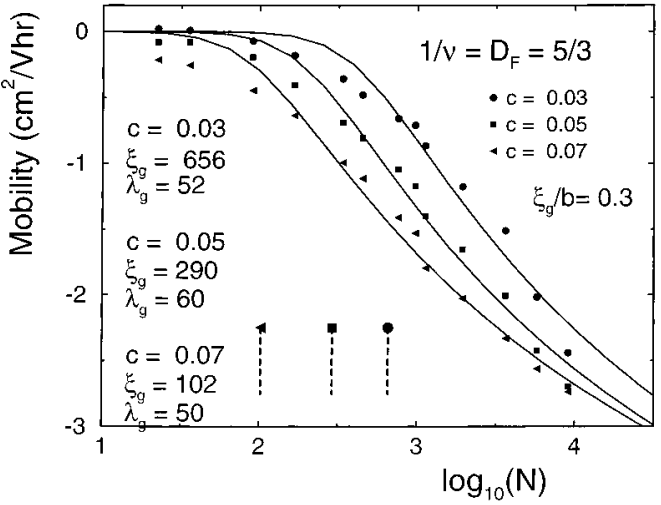

Figure 11. Low-field mobilities of poly(styrenesulfonate) (PSS) tracers in three polyacrylamide (PAA) gels of different concentrations c (wt fraction) versus tracer degree of polymerization, from ref 74 . The full lines are PMC fits with the "soft gel" model, eqs (I.77, I.79, and I.80), with the parameters denoted; gel pore sizes, $\xi_{g}$, are recorded by stating the degree of polymerization of a PSS tracer with identical radii of gyration. The vertical lines mark the tracer degrees of polymerization where $\mathrm{R}_{\mathrm{g}}(\mathrm{N})=\xi_{\mathrm{g}}(\mathrm{c})$.

served than predicted by either Rouse or reptation theory. This has prompted Muthukumar and Baumgärtner to develop the idea of entropic trapping ${ }^{39-42}$ as an alternative transport mechanism that may be dominant in the $\mathrm{R}_{\mathrm{g}} \approx \xi_{\mathrm{g}}$ regime. We will analyze this observation using the finite size effect, namely the constraint porosity, captured by PMC theory for polymer melts and solutions.

As the PAA gel structure is assumed to be similar to the solution structure of PAA, ${ }^{74}$ the fractal dimension appropriate for polymers in good solvents, $D_{F}=5 / 3$ in Flory approximation, is chosen in eq I.75. Also, the soft gel model clearly applies as the cross-linked polymer matrix does not exhibit significantly stronger constraints than the entanglement mesh of the corresponding solution, but only time-independent ones. We use this consideration also to a priori fix the ratio of entanglement length to gel pore size to the value, $\delta=$ $\xi / \mathrm{b}=0.3$, found for solutions in the previous section. Following the suggestion in ref 74 , the mass size scaling of the tracer polymer is also described by the Flory behavior, i.e., $v=3 / 5$ in eq 1.74 .

Figure 11 includes fits with the PMC results, eqs I.79 and 1.80 , for the model of tracer and gel equilibrium structures described above. Least squares optimization was used in order to determine the tracer-gel interaction parameter, $\lambda_{g}$, and the gel pore size, $\xi_{g}$, for three different gel concentrations. It appears physically very reasonable that the tracer-gel interactions are gel density independent, and $\lambda_{\mathrm{g}}=54 \pm 5$ is found for all three densities. Gel pore sizes are reported in Figure 11 by stating the PSS degree of polymerization, $\mathrm{N}$, giving $R_{g}^{\text {PSS }}(N)=\xi_{g}$. The expected decrease of the gel pore size with increasing gel density is clearly observed. A least squares fit to the three data points yields $\xi_{\mathrm{g}} \sim$ $\mathrm{C}^{-1.1}$, which overestimates the expected exponent ${ }^{74}\left(\xi_{\mathrm{g}}\right.$ $\sim \mathrm{c}^{-3 / 4}$ for semidilute good solutions) and lies closer to the $\Theta$ solvent values $\left(\xi_{\mathrm{g}} \sim \mathrm{c}^{-1}\right.$ for semidilute $\Theta$ solutions).

In contrast to the expectations of the entropic barrier model of Muthukumar and Baumgärtner, ${ }^{42}$ the steepest region of the mobility versus mol ecular weight curve is found to lie at $\mathrm{R}_{\mathrm{g}} / \xi_{\mathrm{g}} \gg 1$. The entropic barrier model leads to strongly tracer molecular weight dependent diffusivities when the tracer random coil just fits into the largest gel pores. Although the PMC constraint porosity effects set in when $\mathrm{R}_{\mathrm{g}} \approx \xi_{\mathrm{g}}$, it extends out to very large tracers and leads to high apparent exponents even when $\mathrm{R}_{\mathrm{g}} \gg \xi_{\mathrm{g}}$. Also in contrast to the entropic barrier model, the PMC approach includes the smooth crossovers to the noninteracting Rouse and the asymptotic limits. It not only describes the region of high effective power laws but also describes a smooth variation for all tracer molecular weights. Note that, for the fractal dimensionalities chosen for PSS tracers in PAA gels in Figure 11, the tracer diffusion constants do not crossover to the reptation behavior, $\mu=\mathrm{ND} \sim 1 / \mathrm{N}$, at extremely large tracer molecular weights but instead exhibit the much weaker dependence, $\mu \sim \mathrm{N}^{-2 / 5}$, as follows from eq I.79. This weak asymptotic mol ecular weight dependence is compatible with the data as the crossover to this asymptote is predicted to take place at much higher (unobservable) $\mathrm{N}$ only. In the intermediate range, effective exponents up to $\mu \sim \mathrm{DN} \sim \mathrm{N}^{-2.4}$ have been reported, ${ }^{74}$ which lie in the range and bel ow the upper bound deduced in paper I for these fractal

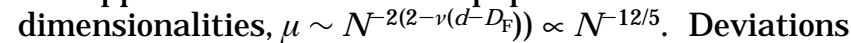
of the fits in Figure 11 for small molecular weights presumably arise from the inadequacy of the Rouse model for short polymers in gels.

Another study of the tracer diffusion of PS through PVME gels $\mathrm{s}^{75}$ was aimed at studying the problem originally considered by de Gennes when introducing the reptation concept..$^{73}$ The gels and their fl exible, neutral polymer solution precursors were well characterized and considerably cross-linked and should therefore be a model system for testing reptation ideas. It was suggested that the gels fall in the light strangulation regime, where the polymer strands between chemical cross-links are somewhat shorter than the ones between the time-dependent entanglements of the non-crosslinked polymer solution. Following the estimates presented in ref 75 we assume that for the three different cross-linking densities studied the length scale ratios, $\xi_{g} / \mathrm{b}$, are somewhat larger than $\delta$ found for solutions. $\xi_{\mathrm{g}} / \mathrm{b}=0.3,0.4$, and 0.5 are chosen as reasonable estimates. The smallest value, found for PS solutions, would correspond to the border of the strangulation regime, and $\xi_{g} / \mathrm{b}=0.5$ to a molecular weight in between cross-links a factor of about 2.8 smaller.

It came as a surprising finding, which was claimed to be in disagreement with reptation theory, that rather strong molecular weight dependences of the PS tracer diffusivities in PVME gels were measured, $D \sim M^{-2.7}$ at gel density $\mathrm{c}=0.200 \mathrm{~g} / \mathrm{mL}$ and $\mathrm{D} \sim \mathrm{M}^{-2.8}$ at $\mathrm{c}=0.235$ $\mathrm{g} / \mathrm{mL}$, respectively. ${ }^{75}$ It was stated that the data agree well with diffusion of PS in PVME solution measurements where larger or equal (in the limit of large matrix molecular weights) diffusivities (also showing anomalous exponents) were found. ${ }^{75}$ Clearly, this aspect of the comparison of tracer motion in solutions and gels is explained by the PMC constraint release mechanism, which is not present in gels but affects the solution data depending on matrix (and tracer) molecular weight. Important for this experimental comparison is the achievement of similar matrix structural correlations in the cases of PVME solutions and gels. ${ }^{75}$ In PMC theory, the constraint porosity effect is important in both solution and gel situations and can explain the finite size fluctuation corrections.

Figure 12 shows PMC model calculations appropriate for the experimentally studied system. As argued above, in order to mimic the gel strangulation regime, the specified values of $\xi_{g} / b$ are chosen. From the results 


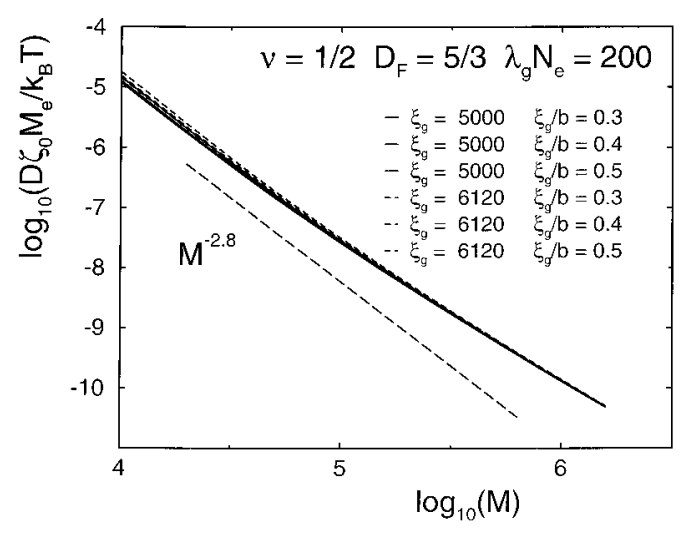

Figure 12. Tracer diffusion constants of polystyrene (PS) as a function of tracer mol ecular weight (M) in poly(vinyl methyl ether) (PVME) gels for parameters comparable to the study in ref 75 calculated from the "soft gel" model, eqs I.77, I.79, and I.80. Gel pore sizes, $\xi_{\mathrm{g}}$, are denoted by the molecular weights of PS tracers with identical radii of gyration. Ratios of pore size to entangl ement length, $\xi / \mathrm{b}$, used are appropriate for polymer solutions, $\xi / \mathrm{b} \approx 0.30$, and lightly strangulated gels, $\xi / \mathrm{b}$ larger.

in Figure 12 it can be seen that small variations with $\xi_{g} / \mathrm{b}$ for the other parameters are obtained. From the stated near identity of the PVME gel and solution density structures, ${ }^{75}$ a Flory excluded volume fractal dimension, $D_{F}=5 / 3$, is deduced for the gels. Screening of the excluded volume interactions of the tracer is assumed for distances larger than the gel pore size; i.e., $v=1 / 2$ is chosen. This choice allows us to connect the gel entanglement strength factor, $\lambda_{\mathrm{g}}$, to the prefactor of the center-of-mass friction function, $\lambda_{\mathrm{D}}$; i.e., $\lambda_{\mathrm{g}}=\lambda_{\mathrm{D}} / \mathrm{N}_{\mathrm{e}}$ follows in eq I.79. In order to crudely account for the known specific charge transfer attractive interactions between PS and PVME, we choose a priori a value $\lambda_{g} N_{e}$ $=200$, which is a factor of about 10 times larger than the value of $\lambda_{D}$ found in PS solutions in section 3.F. Changes of the strength factor $\lambda_{\mathrm{g}}$ by a factor of 2 would not result in appreciable changes of the results in Figure 12. From the results for polymer tracer diffusion in solutions shown in Figure 7 of paper I, one can estimate that for $v=1 / 2$, sol ution-like strength factors could lead to apparent $\mathrm{D}$ versus $\mathrm{N}$ scaling exponents up to 2.6.

Most sensitive for the comparison of the PMC constraint porosity corrections to the measured PS in PVME tracer diffusivities is the ratio of tracer size to gel pore size spanned in the experiments. Following the statement in ref 75, we assume the characteristic pore length of the gel at density $c=0.235 \mathrm{~g} / \mathrm{mL}$ to be $\xi_{\mathrm{g}}=$ $20 \AA$, which agrees with the size of a PS tracer in good solvent for a PS molecular weight of $M=5000$. Note that the experiments span the tracer molecular weight range $10^{4} \leq \mathrm{M} \leq 10^{6}$ and are therefore in the region where $\mathrm{R}_{\mathrm{g}}^{\text {tracer }} \gg \xi_{\mathrm{g}}$. F or the lower gel density, $\mathrm{c}=0.200$ $\mathrm{g} / \mathrm{mL}$, we estimate the PS molecular weight, which leads to $R_{\mathrm{g}}^{\mathrm{PS}}(\mathrm{M})=\xi_{\mathrm{g}}^{\mathrm{PVME}}$ by assuming excluded volume correlations of the gel, $\xi_{\mathrm{g}} \sim \mathrm{C}^{-3 / 4}$, and of the PS tracer inside the gel pores, $\mathrm{R}_{\mathrm{g}} \sim \mathrm{M}^{3 / 5}$ for $\mathrm{R}_{\mathrm{g}} \leq \xi_{\mathrm{g}}$; this leads to $\xi_{\mathrm{g}}(\mathrm{C}=$ $0.200 \mathrm{~g} / \mathrm{mL})$, corresponding to $\mathrm{M}^{\mathrm{PS}}=5000(0.200 /$ $0.235)^{-5 / 4} \approx 6120$.

Figure 12 shows the PMC tracer diffusion coefficients determined with these parameters and thus very roughly corresponding to the experimentally measured PS diffusion coefficients in PVME gels. ${ }^{75}$ Whereas changes of $\lambda_{\mathrm{g}}$ by a factor 2 and $\xi_{\mathrm{g}} / \mathrm{b}$ in the employed range do not change the results appreciably, a larger fractal exponent $v$ of the tracer chain, e.g., $v=3 / 5$, if excluded vol ume for the tracer was not screened, would decrease the slope of the PMC curves in Figure 12 to values around $D \sim N^{-2.2}$. Changes in the fractal dimension of the gel only weakly affect the shape of the PMC curves. For the chosen parameters, much steeper molecular weight dependences than the asymptotic, reptation-like law, result and arise from the spatial correlations of the gel constraints. The tracer polymer feels the full entanglement constraints only if its size considerably exceeds the gel pore size and the length scale, b, of the elastic entanglement mesh. For comparison, a power law $\mathrm{D} \sim \mathrm{N}^{-2.8}$ as reported in ref 75 is shown in Figure 12. The PMC results exhibit somewhat shallower slopes. In evaluating the agreement with the measured tracer diffusivities, one has to consider the reasonable, but ad hoc, estimates of the parameters, the experimental uncertainties, and the complexity of the ternary polymer-solvent-gel system.

\section{Summary and Conclusions}

The picture of the dynamics of entangled polymeric systems as provided by the PMC theory shall be summarized in this final section. A comprehensive overview of all aspects of the PMC approach has been recently given. ${ }^{76}$ The comparisons with experimental data, discussed in the present paper, show that a unified qualitative, and sometimes quantitative, description of the transport properties of entangled polymer chains has been achieved. The comprehensive explanation of a wide variety of experimental observations is noteworthy. Alternative explanations with phenomenological theories, which exist for some of the observed phenomena, fail to consistently explain all of the studied experimental facts. The recent measurements of the dielectric relaxation times of tracer polymers in polymer melts ${ }^{9,10}$ appear to rule out theoretical models which do not consider the collective dynamics of the polymer matrix. Explanations of the anomal ous viscosity versus molecular weight scaling, $\eta \sim \mathrm{M}^{3.5 \pm 0.2}$, provided with models considering a single polymer in a frozen environment, therefore, appear accidental. The same, but frequency-resolved, dielectric measurements ${ }^{9,10}$ stress that the rapid initial decay of the disentanglement process (or its shallow high-frequency loss modulus) persists even in the limit where reptation-like scaling of the final relaxation time is observed. This demonstrates the existence of a nonreptative effect, unrelated to constraint release, which determines the primary disentanglement step. Except for PMC theory, where the tracer shape fluctuations are found responsible, no other consistent theoretical explanation apparently exists for these anomalously shallow high-frequency wings of the dielectric and shear moduli spectra. The measurements of tracer diffusion constants in immobile polymer solutions, i.e., in matrices of high molecular weight polymers ${ }^{26-29}$, and in gels ${ }^{74,75}$ indicate that strong deviations from reptation-like asymptotes can exist even if constraint release corrections are absent. The observation of $a D \sim M^{-2.5}$ scaling in semidilute and concentrated solutions, ${ }^{26-28}$ and of $D \sim M^{-2}$ in melts, 66,77 appears to require a proper theoretical treatment of the polymer liquid structure.

The qualitative picture of the polymer dynamics envisaged by PMC theory is as follows. Entanglements arise from the competition of polymer backbone connectivity and uncrossability due to intermolecular excluded volume forces. Reptation-like asymptotic transport coefficients are obtained for polymers in fixed dense 
environments. This results from the decay of the entanglement friction via the dynamics of the collective single chain structure factor. Two decay channels, a coherent (center-of-mass) one for small wavevectors and an internal one, termed tracer shape fluctuations, for intermediate wavevectors, are associated with the tracer dynamics in the friction functions. The tracer shape fluctuations mainly (greatly) speed up the initial disentanglement process. As entanglement constraints are calculated from the microscopic intermolecular forces, one material dependent strength factor, $1 / \alpha$, enters into the asymptotic PMC predictions. In contrast to the reptation/tube approach where $\alpha$ would be a universal number, in PMC theory $1 / \alpha$ measures the average, mean-squared intermolecular force per unit density and therefore is nonuniversal in principle. Consequently, nonuniversal curves for the relative increase of the transport coefficients, e.g., $\eta / \eta^{\mathrm{R}}$, versus rescaled molecular weight, $\mathrm{n}=\mathrm{N} / \mathrm{N}_{\mathrm{e}}$, are predicted. From comparison with experiments a rather small spread in the values of $\alpha$ for some polymer melts and solutions is found, $\alpha=$ $4 \pm 2$, and the average value lies in the range of the $a b$ initio PRISM estimate.

Finite size corrections to the transport coefficients result in PMC theory from both the decay of the matrix constraints, termed self-consistent constraint release, and from the spatial variations of their amplitudes, called constraint porosity. The spatial dependence of the amplitudes arises from the elastic entanglement mesh, characterized by an entanglement length, b, and the local compressibility correlations, specified by the density screening length or physical mesh, $\xi_{\rho}$. Notethat in PMC theory these effects result from straightforward assumptions about the static structure of the polymeric liquid and its time dependence (see section 3.B of paper I). It can be considered a major achievement of the PMC approach that the variety of non-Rouse and non-reptation-like behaviors of entangled polymer dynamics is derived from one set of microscopically motivated approximations. This approach is very different from ad hoc extensions of the reptation/tube model. There, new types of polymer chain motions are invoked in an empirical and investigator specific fashion, with parameters sometimes of unclear relation to experimental observables, in order to account for unexpected (apparent) exponents characterizing the dependence of a transport coefficient on macromolecular size. This advantage of PMC theory is accompanied by the corre sponding disadvantage that intuitive predictions about the actual monomer trajectories do not naturally emerge.

An important finding of PMC theory relates to the spatial correlations of the entanglement friction in the conformational and the center-of-mass motion. ${ }^{78}$ In conceptual agreement with the basic reptation/tube idea, the center-of-mass motion is determined from rather local friction contributions. The internal dynamics feels constraints that are spatially correlated across the tracer size. A first immediate consequence is the stronger slowing down of the internal dynamics as compared to the translational diffusion. ${ }^{78}$ Another consequence is the very different effects the distinct finite size corrections have on the dynamics. The selfconsistent constraint release mechanism, which is a more effective entanglement force relaxation mechanism than the tracer motion for dynamics on the $R_{g}$ and larger scale, is important for friction contributions on global length scales. The constraint porosity influences the more local friction contributions most strongly.
Therefore, the first finite size effect, constraint release, mainly affects the conformational dynamics, i.e., the internal disentanglement time and the viscosity. The constraint porosity, on the other hand, accelerates the center-of-mass diffusion and the end-to-end vector fluctuations of very long tracers.

The constraint release mechanism is parameterized by the inverse strength factor, $\alpha$, and therefore is rather independent of concentration and solvent quality, as follows from the PRISM estimates. Consequently, PMC theory explains the universal observation of apparent viscosity versus molecular weight power laws, $\eta \sim M^{3.4}$, for both entangled polymer solutions and melts. The observation of a reptation-like scaling of the dielectric relaxation time in matrices of high molecular weight polymers supports the identification of the PMC constraint release mechanism as the origin of the stronger increase of the shear viscosity. Thus, the failure to observe the asymptotic $\mathrm{N}^{3}$ law is predicted to be due to the lack of a clear time scale separation between single chain conformational relaxation and the decay of the entanglement forces. Such a non-Markovian situation has been invoked in the phenomenological "coupling model" picture of Ngai and co-workers ${ }^{79,80}$ and is implicit to the "dynamic cluster" ideas of Douglas and Hubbard. 33

Spatial correlations of the entanglement constraints do not play an important role in polymer melts. As stated and shown in Figures 5 and 6 of paper I, this holds in regard to the internal dynamics for any polymer concentration. If translational diffusion coefficients for fixed reduced degrees of polymerization are considered, the small density screening length in melts again leads to negligible constraint porosity effects. In solutions, however, the center-of-mass mobility is enhanced as the amplitude of the entanglement constraints are proportional to the wavevector-dependent osmotic compressibility. At fixed $n=N / N_{e}$, the density screening length is larger relative to the entanglement length in solutions than in melts. Smaller effective friction coefficients originate from the reduced friction contributions on local length scales.

Crude, a priori PRISM estimates for $\delta=\xi_{\rho} / \mathrm{b}$ lie within a factor of $\approx 5$ from the experimentally found values. In solutions, the magnitude of the uniform PMC friction coefficient extracted from fits, i.e., $\lambda_{D}$ in eqs $I .52$ and I.68, deviates from the prediction, $\lambda_{D}=32 / 3 \alpha$, eq I.53. This deviation is not understood at present, but possibly results from our simplification of a twocomponent polymer-solvent system to an effective, onecomponent polymeric system. Another possible origin is the fundamental PMC approximation, eq I.24, which identifies the decay channels of the entanglement friction forces. The approximation to determine the decay of the entanglement constraints from the collective tracer structure factor, could be qualitatively correct in solutions but not quantitatively accurate. Note that this approximation is supported by our findings for polymer melts. There, a consistent set of parameters describes the translational diffusion coefficient and the viscosity, as shown in section 3.C of this paper.

Another physical situation that indicates the importance of the structural correlations of the matrix entanglements is the polymer tracer motion through a gel. It appears as support of the PMC description of the constraint porosity effects that this common mechanism explains the close connection of tracer diffusivities 
measured in entangled solutions and gels. ${ }^{75}$ In our approach, (soft) gels mainly differ from entangled polymeric matrices in solutions because of the absence of constraint release contributions. Stronger constraint porosity effects than in solutions may arise depending on the fractal dimensionalities of the tracer and the gel, and depending on specific tracer-gel interactions. Whereas the first effects can again be rationalized with simple considerations of the ratio of tracer size to gel pore size, quantitative predictions of the second effects require further experimental and theoretical work on polymer-gel interactions. Strong molecular weight dependences of the tracer diffusivities, e.g., $\mathrm{D} \sim \mathrm{M}^{-2.5}$ in solutions, ${ }^{26-28}$ and $D \sim M^{-2.8}$ in gels, ${ }^{75}$ result from the enhancement of the center-of-mass motion of tracer polymers that do not sample the surrounding entanglement constraints uniformly, as their sizes are not yet asymptotically large.

Finally, we conclude with suggestions for new experiments and/or computer simulations to further test our theoretical ideas about entanglements in linear chain melts, solutions, and gels. The key idea is to manipulate (in a controlled manner) the three fundamental length scales: polymer density (concentration) screening length, $\xi_{\rho}$, entanglement mesh length, b, and tracer radius of gyration, $\mathrm{R}_{\mathrm{g}}$. These nonuniversal structural parameters have a clear physical meaning, are directly measurable, and depend on chemical structure, polymer concentration, temperature, solvent quality, and external pressure. Significant progress in the ability to test our theory against existing and future data, and to make ab initio theoretical predictions, would become possible if there were more direct measurements of the above quantities. This is especially true in $\Theta$ solvents and concentrated solutions (up to the melt) of not only polystyrene but also many other materials. For the problem of tracer motion in gels swollen by solvent, direct measurements of the gel collective structure factor, tracer radius of gyration, and some reliable knowledge of the effective polymer-gel interaction are desirable.

Specific fruitful areas for more experiments include the following. (i) Self-diffusion and tracer diffusion (especially in the effectively frozen matrix limit) of polystyrene in good polystyrene solutions in the 40$100 \%$ polymer concentration regime. Mapping out the concentration and tracer degree of polymerization dependences should allow the observation of the crossover from reptation-like scaling of $D$ in the melt to the "anomalous" solution scaling. Our theory predicts such a crossover and can be quantitatively applied (treating the prefactor $\lambda_{\mathrm{D}}$, which is not understood in solutions, as a free parameter) if the required structural parameters are accurately known. (ii) Same as point i, but in $\theta$ solvents covering the full semidilute-concentratedmelt range. Since the length scale ratio parameter $\delta=$ $\xi_{\rho} / \mathrm{b}$ is larger in $\Theta$ solvents (and more density dependent), we predict enhanced porosity corrections and larger effective $\mathrm{N}$-scaling exponents for the diffusion constant. (iii) points $\mathrm{i}$ and ii for different chemical systems. This is important because even at fixed polymer concentration and solvent quality, the parameter $\delta=\xi_{\rho} / \mathrm{b}$ is nonuniversal ${ }^{56}$ and can potentially be significantly varied. (iv) More tracer measurements in model gels where the probe and matrix (gel) polymers are identical. Alternatively, controlled variations of tracer, solvent, and gel chemical structure could allow the tendency for tracer adsorption or dewetting from the gel structure to be tuned, which in turn is predicted to affect diffusion significantly and in a rationally predictable manner via the parameter $\lambda_{\mathrm{g}}$. (v) Combined selfdiffusion and shear viscosity measurements on variable chemical systems in both the melt and solutions. As usual, the availability of such complementary data allows the monomer friction constant question to be avoided by studying the product $\mathrm{D} \eta$. More importantly, it places additional constraints on our theory by requiring the same set of parameters simultaneously explain the center-of-mass diffusion and conformational (stress) relaxation aspects of entangled dynamics. (vi) Finally, our suggestions ${ }^{60}$ for new experiments at nonzero frequencies, and for transport coefficients, of entangled fluids composed of macromolecules of variable fractal dimensionality (e.g., rings) and in variable spatial dimension. Computer simulation could potentially play a critical role here, although proper treatment of heavily entangled systems remains in the future.

Acknowledgment. Partial financial support by the Deutsche F orschungsgemeinschaft under grant F u 309/ 1-1 and the United States National Science Foundation MRSE C program via grant number NSF -DMR-89-20538 are gratefully acknowledged.

\section{References and Notes}

(1) Fuchs, M.; Schweizer, K. S. Macromolecules 1997, 30, 5133 (preceding article in this issue).

(2) Doi, M. J. Polym. Sci., Polym. Lett. 1981, 19, 265.

(3) Doi, M. J . Polym. Sci., Polym. Phys. Ed. 1983, 21, 667.

(4) Rubinstein, M. Phys. Rev. Lett. 1987, 59, 1946.

(5) O'Connor, N. P. T.; Ball, R. C. Macromolecules 1992, 25, 5677.

(6) Richter, D.; Farago, B.; Fetters, L. J .; Huang, J . S.; Ewen, B.; Lartigue, C. Phys. Rev. Lett. 1990, 64, 1389.

(7) Richter, D.; Willner, L.; Zirkel, A.; Farago, B.; Fetters, L. J .; Huang, J . S. Phys. Rev. Lett. 1993, 71, 4158.

(8) Richter, D.; Willner, L.; Zirkel, A.; Farago, B.; Fetters, L. J .; Huang, J . S. Macromolecules 1994, 27, 7437.

(9) Adachi, K.; Wada, T.; Kawamoto, T.; Kotaka, T. Macromolecules 1995, 28, 3588.

(10) Poh, B. T.; Adachi, K.; Kotaka, T. Macromolecules 1996, 29, 6317

(11) Termonia, Y. Macromol ecules 1996, 29, 2025

(12) Graessley, W. W. Adv. Polym. Sci. 1982, 47, 68.

(13) Klein, J. Macromolecules 1978, 11, 852.

(14) Klein, J. Macromolecules 1986, 19, 105.

(15) des Cloizeaux, J. Europhys. Lett. 1988, 5, 437.

(16) des Cloizeaux, J. Macromol ecules 1990, 23, 4678.

(17) des Cloizeaux, J. Macromolecules 1992, 25, 835.

(18) des Cloizeaux, J . J . Phys. I Fr. 1993, 3, 61.

(19) Baumgaertel, M.; Rosa, M. E. D.; Machado, J .; Masse, M.; Winter, H. H. Rheol. Acta 1992, 31, 75.

(20) J ackson, J .; Rosa, M. E. D.; Winter, H. H. Macromolecules 1994, 27, 2426.

(21) Kannaan, R.; Lodge, T. P. Macromolecules 1997, 30, 3694.

(22) Adachi, K.; Kotaka, T. Macromolecules 1985, 18, 466.

(23) Imanishi, Y.; Adachi, K.; Kotaka, T. J . Chem. Phys. 1988, 89, 7585.

(24) Adachi, K.; Yoshida, H.; Fukui, F.; Kotaka, T. Macromol ecules 1990, 23, 3138

(25) Watanabe, H.; Tirell, M. Macromolecules 1989, 22, 927.

(26) Nemoto, N.; Kojima, T.; Inoue, T.; Kishine, M.; Hirayama, T.; Kurata, M. Macromolecules 1989, 22, 3793.

(27) Nemoto, N.; Kishine, M.; Inoue, T.; Osaki, K. Macromolecules 1990, 23, 659 .

(28) Nemoto, N.; Kishine, M.; I noue, T.; Osaki, K. Macromolecules 1991, 24, 1648.

(29) Nemoto, N. In Polymer Rheol ogy and Processing; Collyer, A. A., Utracki, L. A.,, Eds.; Elsevier Science Publishers Ltd.; London and New York, 1990, p 3.

(30) Deutsch, J . M.; Madden, T. L. J . Chem. Phys. 1989, 91, 3252.

(31) Rubinstein, M.; Obukhov, S. P. Phys. Rev. Lett. 1993, 71, 1856.

(32) Fixman, M. J . Chem. Phys. 1988, 89, 3892.

(33) Douglas, J . F.; Hubbard, J. B. Macromolecules 1991, 24, 3163.

(34) Herman, M. F. J . Chem. Phys. 1990, 89, 3892. 
(35) Herman, M. F.; Panajotova, B.; Lorenz, K. T. J . Chem. Phys. 1996, 105, 1153.

(36) Loring, R. F. J . Chem. Phys. 1991, 94, 1505.

(37) Chatterjee, A. P.; Loring, R. F. J . Chem. Phys. 1995, 103, 4711.

(38) Chatterjee, A. P.; Geissler, P. L.; Loring, R. F. J . Chem. Phys. 1996, 104, 5284.

(39) Baumgärtner, A.; Muthukumar, M. J . Chem. Phys. 1987, 87, 3082.

(40) Muthukamar, M.; Baumgärtner, A. Macromol ecules 1989, 22, 1937.

(41) Muthukumar, M.; Baumgärtner, A. Macromol ecules 1989, 22 , 1941.

(42) Baumgärtner, A.; Muthukumar, M. Adv. Chem. Phys. 1996, 94,625

(43) Zimm, B. H.; Lumpkin, O. Macromolecules 1993, $26,226$.

(44) Schweizer, K. S.; Szamel, G. J . Chem. Phys. 1995, 103, 1934.

(45) Schweizer, K. S.; Szamel, G. Macromolecules 1995, 28, 7543.

(46) Fetters, L. J .; Lohse, D. J .; Richter, D.; Witten, T. A.; Zirkel, A. Macromolecules 1994, 27, 4639.

(47) Brown, W.; Nicolai, T. Colloid Polym. Sci. 1990, 268, 977.

(48) Doi, M.; Edwards, S. F. The Theory of Polymer Dynamics; Oxford University Press: Oxford, U.K., 1986.

(49) Colby, R. H.; Rubinstein, M. Macromolecules 1990, 23, 2753.

(50) Pearson, D. S. Rubber Chem. Technol. 1987, 60, 437.

(51) Schweizer, K. S.; Curro, J. G. Adv. Polym. Sci. 1994, 116, 319.

(52) Schweizer, K. S.; Curro, J . G. Adv. Chem. Phys. 1996, 98, 1.

(53) Schweizer, K. S.; Curro, J. G. Chem. Phys. 1990, 149, 105.

(54) Hansen, J. P.; McDonald, I. R. Theory of Simple Liquids; Academic Press: London, 1986.

(55) Schweizer, K. S.; Curro, J. G. Phys. Rev. Lett. 1987, 58, 246

(56) Raspaud, E.; Lairez, D.; Adam, M. Macromol ecules 1995, 28, 927.

(57) Paul, W.; Binder, K.; Heermann, D. W.; Kremer, K. J . Phys. II (Paris) 1991, 1, 37.

(58) Paul, W.; Binder, K.; Heermann, D. W.; Kremer, K.J . Chem. Phys. 1991, 95, 7726.
(59) David, E. F.; Schweizer, K. S. Unpublished.

(60) Fuchs, M.; Schweizer, K. S. J . Chem. Phys. 1997, 106, 347.

(61) Colby, R. H.; Fetters, L. J .; Graessley, W. W. Macromolecules 1987, 20, 2226

(62) Pearson, D. S.; Fetters, L. J .; Graessley, W. W.; Strate, G. V.; von Meerwall, E. Macromolecules 1994, 27, 711.

(63) Fleischer, G.; Appel, M. Macromolecules 1995, 28, 7281.

(64) Appel, M.; Fleischer, G. Macromolecules 1993, 26, 5520.

(65) Antonietti, M.; Fölsch, K. J .; Sillescu, H. Makromol. Chem. 1987, 188, 2317.

(66) Green, P. F.; Kramer, E. J . Macromolecules 1986, 19, 1108.

(67) Roovers, J. Polymer 1985, 26, 1091.

(68) Plazek, D. Private communication. American Physical Society Meeting, New York, 1987.

(69) Stockmayer, W. H. Pure Appl. Chem. 1967, 15, 539.

(70) Schweizer, K. S.; Szamel, G. Trans. Theo. Stat. Phys. 1995 $24,947$.

(71) Schweizer, K. S.; Szamel, G. J . Chem. Phys., to be submitted for publication.

(72) Ferry, J . D. Viscoelastic Properties of Polymers; Wiley: New York, 1980.

(73) de Gennes, P. G. J . Chem. Phys. 1971, 55, 572.

(74) Arvanitidou, E.; Hoagland, D. Phys. Rev. Lett. 1991, 67, 1464.

(75) Rotstein, N. A.; Lodge, T. P. Macromol ecules 1992, 25, 1316.

(76) Schweizer, K. S.; Fuchs, M.; Szamel, G.; Guenza, M.; Tang, H. Macromolecules Theory and Simulation, in press, 1997.

(77) Green, P. F.; Mills, P. J .; Palmstrom, C. J .; Mayer, J . W.; Kramer, E. J . Phys. Rev. Lett. 1984, 58, 2145.

(78) Schweizer, K. S. J . Chem. Phys. 1989, 91, 5822.

(79) Ngai, K. L.; Rajagopal, A. K.; Tietler, S.J . Chem. Phys. 1988 $88,5086$.

(80) Ngai, K. L.; Skolnick, J. Macromolecules 1991, 24, 1561.

(81) Flory, P. J . Principles of Polymer Chemistry; Cornell University Press: Ithaca, NY, 1953.

MA9702354 